\title{
The 3-D ionization structure of NGC 6818: A Planetary Nebula threatened by recombination ${ }^{\star}$
}

\author{
S. Benetti ${ }^{1}$, E. Cappellaro ${ }^{2}$, R. Ragazzoni ${ }^{3}$, F. Sabbadin ${ }^{1}$, and M. Turatto ${ }^{1}$ \\ 1 INAF - Osservatorio Astronomico di Padova, vicolo dell'Osservatorio 5, 35122 Padova, Italy \\ 2 INAF - Osservatorio Astronomico di Capodimonte, via Moiariello 11, 80131 Napoli, Italy \\ ${ }^{3}$ INAF - Osservatorio Astrofisico di Arcetri, Largo E. Fermi 5, 50125, Italy
}

Received 24 September 2002 / Accepted 11 November 2002

\begin{abstract}
Long-slit NTT+EMMI echellograms of NGC 6818 (the Little Gem) at nine equally spaced position angles, reduced according to the 3-D methodology introduced by Sabbadin et al. (2000a,b), allowed us to derive: the expansion law, the diagnostics and ionic radial profiles, the distance and the central star parameters, the nebular photo-ionization model, the 3-D reconstruction in He II, [O III] and [N II], the multicolor projection and a series of movies. The Little Gem results to be a young (3500 years), optically thin (quasi-thin in some directions) double shell ( $M_{\mathrm{ion}} \simeq 0.13 M_{\odot}$ ) at a distance of $1.7 \mathrm{kpc}$, seen almost equatorial on: a tenuous and patchy spherical envelope $(r \simeq 0.090 \mathrm{pc})$ encircles a dense and inhomogeneous tri-axial ellipsoid $(a / 2 \simeq 0.077 \mathrm{pc}, a / b \simeq 1.25, b / c \simeq 1.15)$ characterized by a hole along the major axis and a pair of equatorial, thick moustaches. NGC 6818 is at the start of the recombination phase following the luminosity decline of the $0.625 M_{\odot}$ central star, which has recently exhausted the hydrogen shell nuclear burning and is rapidly moving toward the white dwarf domain $\left(\log T_{*} \simeq 5.22 \mathrm{~K}\right.$; $\log L_{*} / L_{\odot} \simeq 3.1$. The nebula is destined to become thicker and thicker, with an increasing fraction of neutral, dusty gas in the outermost layers. Only over some hundreds of years the plasma rarefaction due to the expansion will prevail against the slower and slower stellar decline, leading to a gradual re-growing of the ionization front. The exciting star of NGC $6818\left(m_{V} \simeq 17.06\right)$ is a visual binary: a faint, red companion $\left(m_{V} \simeq 17.73\right)$ appears at $0.09 \operatorname{arcsec}$ in $\mathrm{PA}=190^{\circ}$, corresponding to a separation $\geq 150 \mathrm{AU}$ and to an orbital period $\geq 1500$ years.
\end{abstract}

Key words. planetary nebulae: individual: NGC 6818 - ISM: kinematics and dynamics

\section{Introduction}

It is widely accepted that an "aged" star of low to medium mass $\left(1.0 M_{\odot}<M_{\mathrm{MS}}<8.0 M_{\odot}\right)$ which is evolving toward the white dwarf region of the $\mathrm{H}-\mathrm{R}$ diagram, first expels and then ionizes the surface layers, thus generating the Planetary Nebula (PN) phenomenology (Paczynski 1970; Aller 1984; Pottasch 1984; Osterbrock 1989).

Thanks to the advent of sophisticate photo-ionization codes (Harrington 1989; Pequignot 1997; Ferland et al. 1998), we now know in detail the physical effects produced by any UV

Send offprint requests to: $\mathrm{S}$. Benetti,

e-mail: benetti@pd.astro.it

* Based on observations made with ESO Telescopes at the La Silla Observatories, under programme ID 65.I-0524, and on observations made with the NASA/ESA Hubble Space Telescope, obtained from the data archive at the Space Telescope Institute (observing programs GO 7501 and GO 8773; P.I. Arsen Hajian). STScI is operated by the association of Universities for Research in Astronomy, Inc. under the NASA contract NAS 5-26555. We have applied the photoionization code CLOUDY, developed at the Institute of Astronomy of the Cambridge University. flux on any gas distribution and composition. Conversely, the situation is quite disappointing for a true nebula, due to projection limitations: on the one hand the HST imagery has strongly enhanced the ground-based evidences of the PNe complexity, on the other hand the observational data are still interpreted in terms of approximate structures and unrealistic assumptions for the physical conditions, like $\mathrm{Te}=$ constant and $\mathrm{Ne}=$ constant all over the object (more comments are in Aller 1984, 1990, 1994).

In order to overcome the wide gap between theory and practice, the apparent, bi-dimensional nebular image should be deprojected, and the accurate spatial distribution of the gas recovered.

To this end we have developed an original procedure based on high dispersion spectra: the PN being an extended and expanding plasma, the position, thickness and density of each elementary volume can be, in principle, obtained from the radial velocity, width and flux of the corresponding emission. We first apply a tomographic analysis, which reconstructs the ionic distribution in the nebular slices covered by the spectrograph slit, and then assemble all the tomographic maps by means of a 


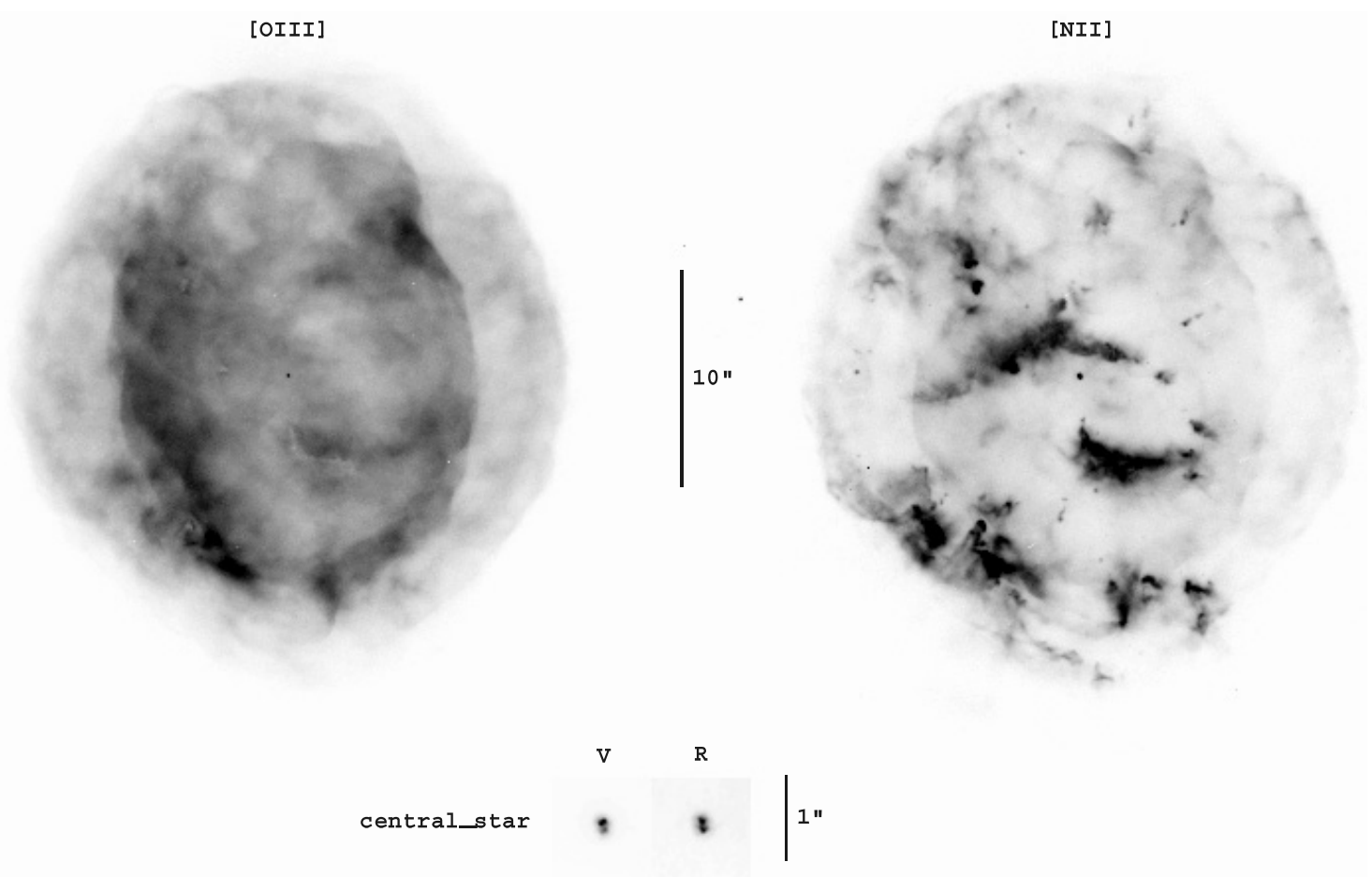

Fig. 1. WFPC2 archive images of NGC 6818 in [O III] (left) and [N II] (right). The binary nature of the central star is shown in the enlargement at the bottom. North is up and East to the left.

3-D rendering procedure for studying the morphology, physical conditions, ionization, spatial structure and evolutionary status.

The rationale of the method and the earliest, rough results based on plate echellograms go back to Sabbadin et al. (1985, 1987). More recent, "quantitative" observations (i.e. using a linear detector) concern NGC 40 and NGC 1501, both objects covered at moderate spectral resolution, $R \simeq 20000-25000$, with the Echelle $+1.82 \mathrm{~m}$ telescope of Padua Observatory at Asiago, Cima Ekar (Sabbadin et al. 2000a,b; Ragazzoni et al. 2001; hereafter Papers I to III, respectively).

At the same time we have carried out a survey of two dozen PNe and proto-PNe in both hemispheres with ESO NTT+EMMI (spectral range $\lambda \lambda 3900-7900 \AA, R=60000$, spatial resolution $\simeq 1.0$ arcsec) and the Telescopio Nazionale Galileo (TNG)+SARG (spectral range $\lambda \lambda 4600-8000 \AA, R=$ 115000 , spatial resolution $\simeq 0.7$ arcsec). The observed sample covers a variety of morphologies, kinematics and evolutionary phases, including NGC 2392 (the Eskimo nebula), NGC 3132 (the Eight-Burst n.), NGC 3242 (the Ghost of Jupiter n.), NGC 6210 (the Turtle n.), NGC 6543 (the Cat's Eye n.), NGC 6751 (the Glowing Eye n.), NGC 6826 (the Blinking n.), NGC 7009 (the Saturn n.), NGC 7662 (the Blue Snowball n.), IC 418 (the Spirograph n.), He 2-47 (the Starfish n.), MyCn 18 (the Hourglass n.), MZ 3 (the Ant n.), and as many un-dubbed, but equally exciting targets.

The 3-D ionization structure of NGC 6565 has been presented by Turatto et al. (2002, Paper IV); here we discuss the case of NGC 6818 (PNG 025.8-17.9, Acker et al. 1992).

The paper is structured as follows: Sect. 2 introduces the nebula, Sect. 3 presents the observational material and the reduction procedure, Sect. 4 is dedicated to the gas kinematics,
Sect. 5 concerns the radial profile of the physical conditions (electron temperature and electron density from forbidden line ratios), in Sect. 6 the ionization structure and the overall gas distribution are discussed, in Sect. 7 we derive the nebular distance, mass and age, in Sect. 8 the central star parameters are given, Sect. 9 contains the application of the photo-ionization model (CLOUDY), Sect. 10 describes the 3-D structure of the nebula in different ions, and Sect. 11 presents a short discussion and the conclusions.

\section{The nebula}

The HST/WFPC2 appearance of the high excitation PN NGC 6818 (sometimes called the Little Gem) is shown in Fig. 1: in [O III] "a roughly spherical outer envelope as well as a brighter vase-shaped interior bubble. There is a prominent orifice to the North and a smaller one to the South, along the major axis, probably caused by a blow-out from a fast wind" (Rubin et al. 1998). A few dark filaments and knots are also present.

The [N II] emission mainly occurs in two irregular equatorial "moustaches" and in a multitude of condensations (in some cases presenting a sort of radially arranged cometary tail), which are brighter in the southern part of the nebula.

The striking HST multicolor reproduction by Arsen Hajian \& Yervant Terzian (http://ad.usno.navy.mil/ pne/gallery.html) highlights the composite envelope structure and the large stratification of the radiation within NGC 6818.

Rubin et al. (1998) reported the presence of two faint stars, roughly $2-4 \operatorname{arcsec} \mathrm{N}$ and $\mathrm{NE}$ of the central star, which 
could be physically associated to it. A careful analysis of the WFPC2 frames allowed us to unveil the true binary nature of the central star: the red, faint companion is at a distance of $0.093( \pm 0.004)$ arcsec in position angle $(\mathrm{PA})=190( \pm 2)^{\circ}($ see the enlargement at the bottom of Fig. 1).

Following Weedman (1968) our nebula is a prolate spheroid (an ellipse rotated about the major axis) of moderate ellipticity (axial ratio 3:2) observed perpendicular to the major axis, whereas Sabbadin (1984) suggests a somewhat optically thick prolate spheroid in a early evolutionary phase, seen almost pole-on. According to Hyung et al. (1999), a definitive statement on the geometry must wait for more adequate kinematical data, due to the complexity of NGC 6818 .

To this end we have secured a series of long-slit, radially arranged echellograms. They were analysed with the 3-D methodology described in Papers I to IV.

\section{The observational material and the reduction technique}

Nine echellograms of NGC 6818 (exposure time 600 s; spectral resolution 60000 with a slit 1.0 arcsec wide) have been obtained on July 29, 2000 at the ESO NTT in photometric sky conditions and seeing between 0.7 and 1.0 arcsec. We have used a 40 arcsec long slit centered on the exciting star for all the selected PA, ranging from $10^{\circ}$ to $170^{\circ}$ with a constant step of $20^{\circ}$. Since we do not insert an interference filter (as normally done by the other observers to isolate a single order), each spectrum covers 80 echelle orders ( $\lambda 3967 \AA$ of $[\mathrm{Ne}$ III] to $\lambda 7751 \AA$ of [Ar III]), and provides the spatio-kinematical structure of a good two dozen nebular emissions, representing all the main ionic species.

The reduction method follows conceptually the standard procedure, including bias, flat field, distortion correction, wavelength and flux calibration, and is carefully described in Paper IV.

Figure 2 illustrates the detailed structure in [O I] $(\lambda 6300 \AA)$, [N II] $(\lambda 6584 \AA)$, H I ( $\lambda 6563 \AA)$, [O III] ( $25007 \AA)$ and He II $(\lambda 4686 \AA)$ at the observed PA. NGC 6818 exhibits a complex ionization structure:

- $[\mathrm{O} \mathrm{I}]$ is only seen in the outermost regions, in the form of distinct condensations, which in some cases are symmetrically arranged (the moustaches), otherwise single (like the cometary knot in $\mathrm{PA}=150^{\circ}$, southern edge);

- the low ionization [N II] emission forms a double envelope of inhomogeneous structure from $\mathrm{PA}=50^{\circ}$ to $\mathrm{PA}=130^{\circ}$, and a single, elongated and distorted ring from $\mathrm{PA}=150^{\circ}$ to $\mathrm{PA}$ $=30^{\circ}$. The presence of FLIERS (fast, low ionization emitting regions), as introduced by Balick et al. (1993, see also Corradi et al. 1996), is not confirmed here: although some isolated spikes present a faint, high velocity tail, the overall [N II] emission of NGC 6818 can be understood in terms of "normal" nebular regions simply shadowed by some inner and dense layer causing the ionization drop in the outer plasma;

- the $\mathrm{H} \alpha$ line (central row in Fig. 2) is characterized by a very blurred appearance (due to a combination of thermal motions plus fine structure plus expansion velocity gradient), masking the detailed distribution of the ionized gas. The same effect is also present in He I and He II, but it is particularly damaging for $\mathrm{H} \alpha$, the reference emission in both the radial electron density and ionization determinations (Paper IV);

- $\lambda 5007 \AA$ of [O III] (a mean-high excitation ion), by far the strongest line in the optical region, highlights the double envelope structure of NGC 6818: the inner shell (better seen between $\mathrm{PA}=50^{\circ}$ and $\mathrm{PA}=130^{\circ}$ ) is an irregular ellipsoid broken along the major axis (N-S direction) whose equatorial, denser regions are identified by the "moustaches". The line-tilt between $\mathrm{PA}=90^{\circ}$ and $\mathrm{PA}=110^{\circ}$ suggests that we are misaligned with both the intermediate and the minor axes of the inner ellipsoid, and that the line of the nodes is at $\mathrm{PA} \simeq 60^{\circ}$. The outer shell is almost un-tilted, spherical, holed at North and South, and circumscribes the internal one;

- the He II emission (bottom row in Fig. 2) marks the highest excitation nebular regions, mainly constituted by the inner shell. Note the blurred appearance of $\lambda 4686 \AA$, essentially due to the thirteen fine structure components.

The first, qualitative picture of the spatial structure coming from the echellograms confirms the indications already obtained from the imaging (Sect. 2).

\section{The gas kinematics}

According to Papers I to IV, the expansion velocity (Vexp) of the ionized gas can be derived from the analysis of the "central star pixel line" (cspl) in the different ions. The cspl is parallel to the dispersion, selects the nebular material projected at the apparent position of the star (whose motion is purely radial) and is the same in all the frames, the slit being radially arranged. Thus, in order to improve the $\mathrm{S} / \mathrm{N}$ of the faintest emissions, the nine echellograms have been combined.

The results are contained in the last column of Table 1, where the ions are put in order of increasing ionization potential (IP). Typical errors are $1.5 \mathrm{~km} \mathrm{~s}^{-1}$ for the strongest forbidden emissions (like $\lambda 6584 \AA$ of [N II] and $\lambda 5007 \AA$ of [O III]) to $3.0 \mathrm{~km} \mathrm{~s}^{-1}$ for the faintest ones (in particular: $\lambda 6300 \AA$ of [O I], because of the knotty structure, and $\lambda 7319.87 \AA$ of [O II], which is partially blended with $\lambda 7318.79 \AA$ also belonging to the $\mathrm{O}^{+}$red quartet). The corresponding uncertainties for the recombination lines are: $2.5 \mathrm{~km} \mathrm{~s}^{-1}$ for $\lambda 6563 \AA(\mathrm{H} \mathrm{I})$, and $2.0 \mathrm{~km} \mathrm{~s}^{-1}$ for $\lambda 5876 \AA$ (He I) and $\lambda 4686 \AA$ (He II).

Columns 3 to 6 of Table 1 report the kinematical results from the literature. In detail:

- Wilson (1950) obtained a single Coudè spectrum of NGC 6818 (without de-rotator) at spectral resolution $R \simeq$ 30000 ;

- Sabbadin (1984) observed the nebula at four PA (long-slit echellograms at $R \simeq 15000$ );

- Meatheringham et al. (1988, long-slit echellograms at unspecified position, $R \simeq 26000$ ) also measured the width at $10 \%$ maximum intensity of $\lambda 5007 \AA$ obtaining $2 \mathrm{~V} \exp [\mathrm{O}$ III] $=$ $87.6 \mathrm{~km} \mathrm{~s}^{-1}$ (following Dopita et al. 1985, this corresponds to the largest expansion velocity of the gas);

- Hyung et al. (1999, $R \simeq 33000$ ) studied four bright regions located along the apparent minor and major axes (4-5 arcsec East and West, and 9-10 arcsec North and South of the central 


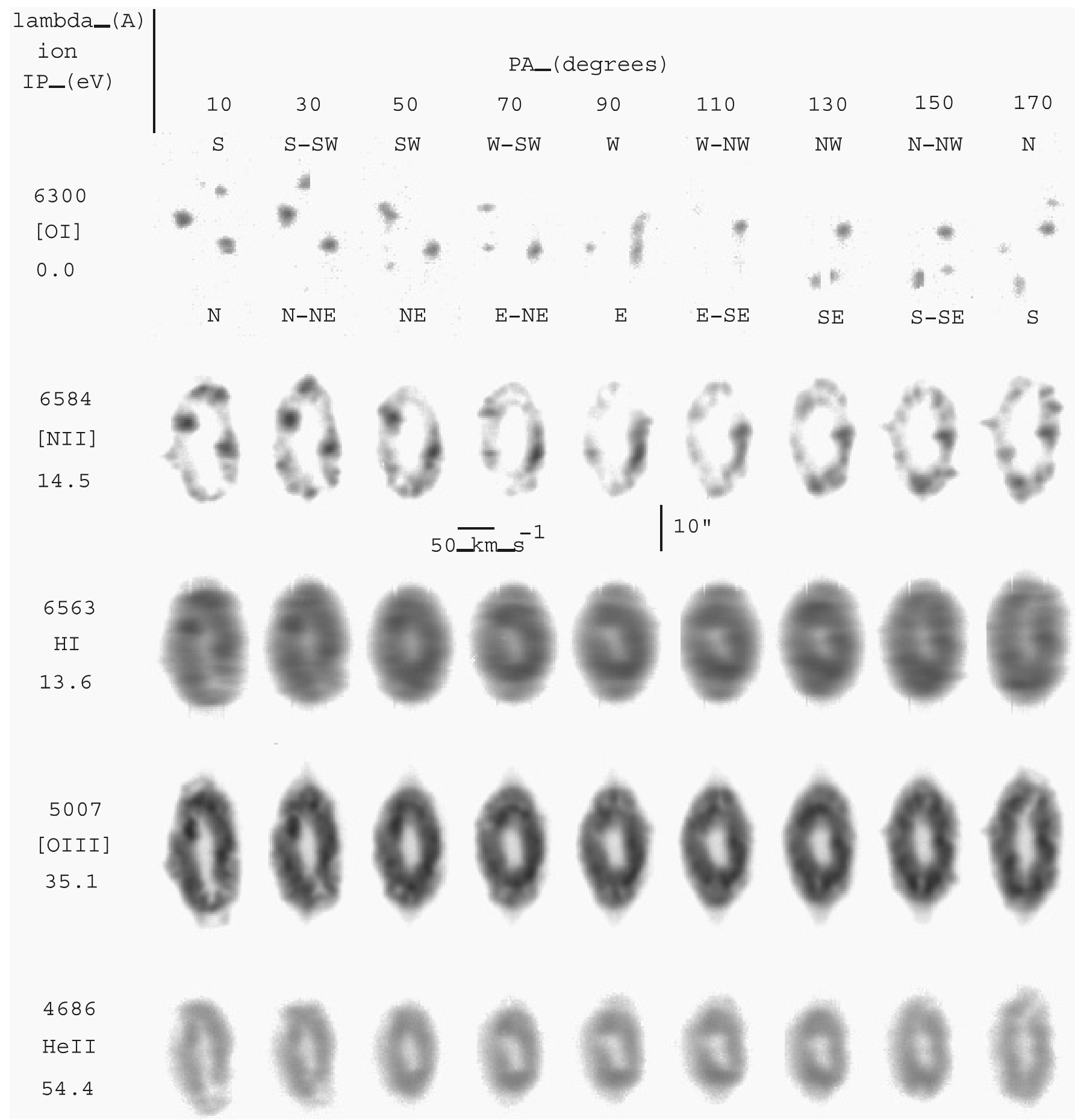

Fig. 2. Detailed structure (in logarithmic scale) of some representative emissions at the nine observed PA of NGC 6818. The blue-shifted gas is to the left. The slit orientation at each PA is indicated in the corresponding [O I] frame. The $\lambda 6300.304 \AA$ night sky line has been removed from the $[\mathrm{O} I]$ spectral image.

star); their values represent lower limits to $V \exp ($ various ions), due to projection effects.

In Table 1 the kinematical variety reported by the different authors is symptomatic of the difficulties connected to the analysis of the high dispersion spectra, and, according to Hyung et al. (1999), stresses the fact that only the detailed coverage at adequate spatial and spectral resolutions can provide a reliable information on the structure of a chaotic object like NGC 6818.

As cspl refers to the kinematical properties of the matter projected at the apparent position of the star, so the "zero velocity pixel column" (zvpc), corresponding to the recession 
Table 1. Peak separation in the cspl of NGC 6818.

\begin{tabular}{|c|c|c|c|c|c|c|}
\hline \multirow[t]{2}{*}{ Ion } & \multirow[t]{2}{*}{$\mathrm{IP}(\mathrm{eV})$} & \multicolumn{5}{|c|}{ 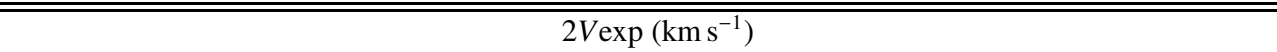 } \\
\hline & & Wilson (1950) & Sabbadin (1984) & Meatheringham et al. (1988) & Hyung et at. (1999) & this paper \\
\hline$[\mathrm{O} I]$ & 0.0 & - & - & - & - & 72: \\
\hline [S II $]$ & 10.4 & - & - & - & 60.7 & 71 \\
\hline [O II $]$ & 13.6 & 60.2 & - & 59.1 & 58.9 & 69: \\
\hline H I & 13.6 & 55.5 & 52 & - & 42.6 & 55 \\
\hline$[\mathrm{N}$ II $]$ & 14.5 & - & 64 & - & 61.8 & 70 \\
\hline [S III $]$ & 23.4 & - & - & - & 50.8 & 65: \\
\hline $\mathrm{He} \mathrm{I}$ & 24.6 & - & - & - & 57.3 & 66 \\
\hline [Ar III] & 27.6 & - & - & - & 48.9 & 64 \\
\hline [O III] & 35.1 & 56.2 & 54 & 55.1 & 51.3 & 62 \\
\hline [Ar IV] & 40.7 & - & - & - & 30.7 & 48 \\
\hline [Ne III] & 41.0 & 58.0 & - & - & 52.2 & 63 \\
\hline N III & 47.4 & - & - & - & 41.5 & - \\
\hline He II & 54.4 & 42.4 & - & 41.9 & 31.5 & 45 \\
\hline [Ar V] & 59.8 & - & - & - & 20.2 & 36 \\
\hline$[\mathrm{Ne} \mathrm{V}]$ & 97.1 & 32.6 & - & - & - & - \\
\hline
\end{tabular}

velocity of the whole nebula, gives the radial distribution of the ionized gas which is expanding perpendicularly to the line of sight (Paper IV and references therein).

The intensity peak separations, $2 r_{\mathrm{zvpc}}$, in the different emissions at the nine PA of NGC 6818 are presented in Table 2. Both Fig. 2 and Table 2 indicate complex zvpc profiles, often multi-peaked (inner and outer shell), fast changing in direction, and variable (at a given PA) from ion to ion. The observed line structure depends on the radial matter distribution, the ionization and the intensity of the emission (the same occurs for the cspl assuming $V \exp \propto r$, as normally observed in $\mathrm{PNe}$ ).

To be noticed: the "relative" spectral and spatial resolutions of our echellograms, as introduced in Paper III, differ by a factor of two. The former is given by $R R=V \exp / \Delta V \simeq 6, \Delta V$ being the spectral resolution, and the latter by $S S=r / \Delta r \simeq$ $12(r=$ apparent radius, $\Delta r=$ seeing). In practice this means that the spatial information of NGC 6818 is twice as detailed as the kinematical one.

In order to assemble the kinematical and the spatial results (corresponding to a single radial direction and to nine tangential directions, respectively) we must identify the PA at which $r_{\mathrm{zvpc}} \simeq r_{\mathrm{cspl}}$. The solution comes from the qualitative picture resulting from both the imaging (Sect. 2) and the spectra (Sects. 3 and 4). Let's consider for the main component of NGC 6818 the most general spatial structure, i.e. a tri-axial ellipsoid. The major axis, projected in $\mathrm{PA} \simeq 10^{\circ}$, is almost perpendicular to the line of sight, since the emissions, although inhomogeneous and distorted by irregular motions, appear un-tilted. The intermediate and the minor axes lie in $\mathrm{PA} \simeq 100^{\circ}$, and the line-tilt observed at $\mathrm{PA}=90^{\circ}$ and $110^{\circ}$ indicates that we are misaligned with both these axes. Thus, $r_{\mathrm{zvpc}} \simeq r_{\mathrm{cspl}}$ along the apparent minor axis of the nebula, that is between $\mathrm{PA}=90^{\circ}$ and $110^{\circ}$.

The cspl-zvpc connection for the inner and the outer shells is illustrated in Fig. 3, showing both $r_{\mathrm{zvpc}}$ (at $\mathrm{PA}=90^{\circ}$ and $110^{\circ}$ ) and $V \exp$ vs. IP. For reasons of homogeneity each zvpc peak separation is normalized to the corresponding [O III] value.

NGC 6818 is characterized by a large stratification of the radiation and of the kinematics, in agreement with the Wilson's (1950) law. However, Vexp and $r_{\mathrm{zvpc}}$ of [Ar IV] and [Ne III] considerably deviate from the sequence defined by the other ionic species. This behavior, also noticed in NGC 6565 (Paper IV), is typical of a PN powered by a high temperature central star (for details, see the above-mentioned reference and Sect. 9). Instead, the anomalous position of H I in Fig. 3 is the obvious consequence of the mono-electron atomic structure of hydrogen.

The $r_{\text {zvpc }}$ vs. IP relation being the same at all the observed PA (from Table 2), we can assess that in NGC 6818 the expansion velocity is proportional to the distance from the central star through the relation:

$V \exp \left(\mathrm{km} \mathrm{s}^{-1}\right)=3.5( \pm 0.3) \times r^{\prime \prime}$.

Weedman (1968) has derived $V \exp \left(\mathrm{km} \mathrm{s}^{-1}\right)=5.9 \times\left(r^{\prime \prime}-0.8\right)$, which is almost twice as steep as Eq. (1). The Weedman's expansion law appears questionable, since it is based on the [O III] profile in a single, quite under-exposed Coudè spectrum $(R \simeq 60000)$ taken along the apparent major axis $\left(\mathrm{PA} \simeq 10^{\circ}\right)$. Weedman noticed that $\lambda 5007 \AA$, although distorted by irregular motions, is un-tilted, and assumed "a priori" the following model: a prolate spheroid with $a=17.3 \operatorname{arcsec}$ and $a / b=1.5$, seen perpendicular to the major axis.

Therefore in the following we will consider Eq. (1) as representative of the whole nebular kinematics.

All this is synthesized in Fig. 4, showing the positionvelocity $(P-V)$ maps, i.e. the complete radial velocity field at the nine observed PA. They are relative to the systemic heliocentric velocity of the nebula, $V r_{\odot}=-14.5( \pm 1.0) \mathrm{km} \mathrm{s}^{-1}$, corresponding to $V_{\mathrm{LSR}}=-1.7( \pm 1.0) \mathrm{km} \mathrm{s}^{-1}$, and are scaled 
Table 2. Peak separation in the zvpc at the nine observed PA of NGC 6818.

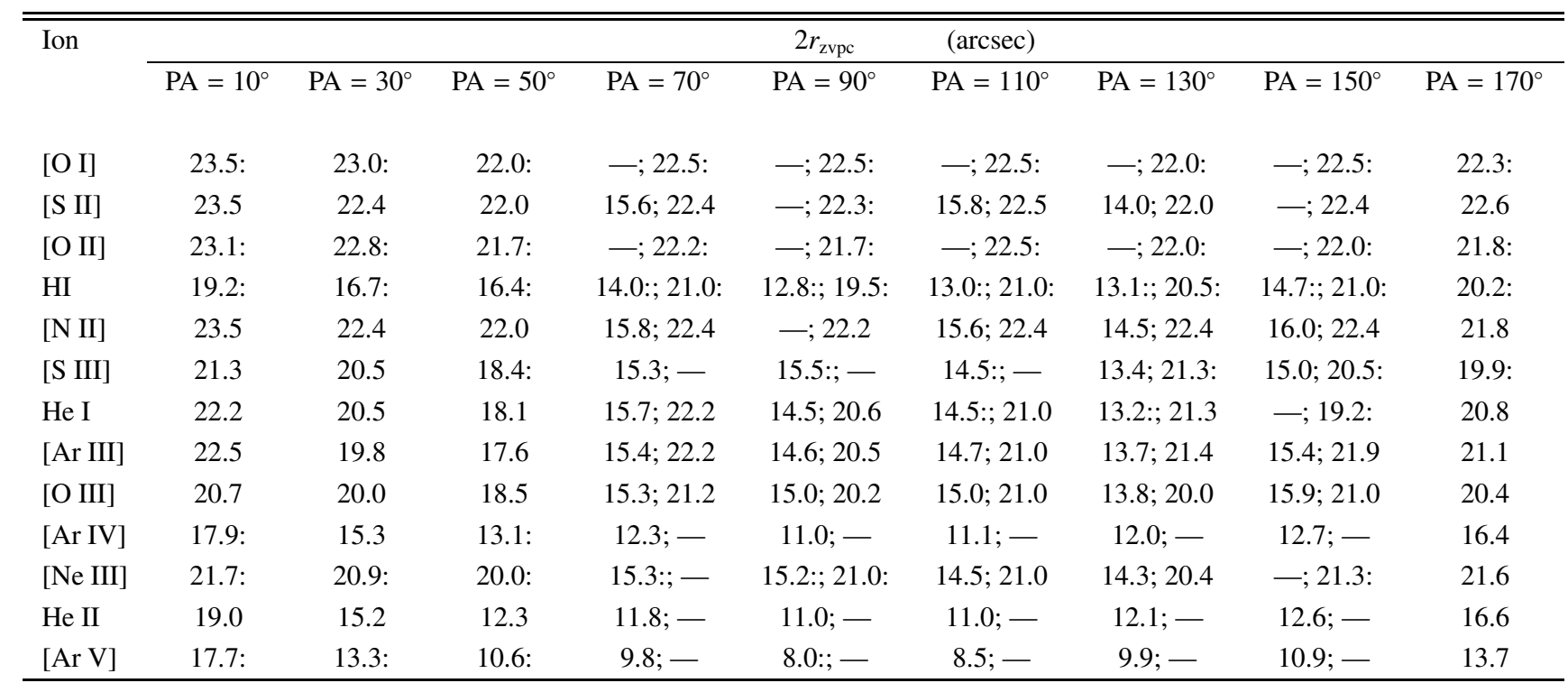

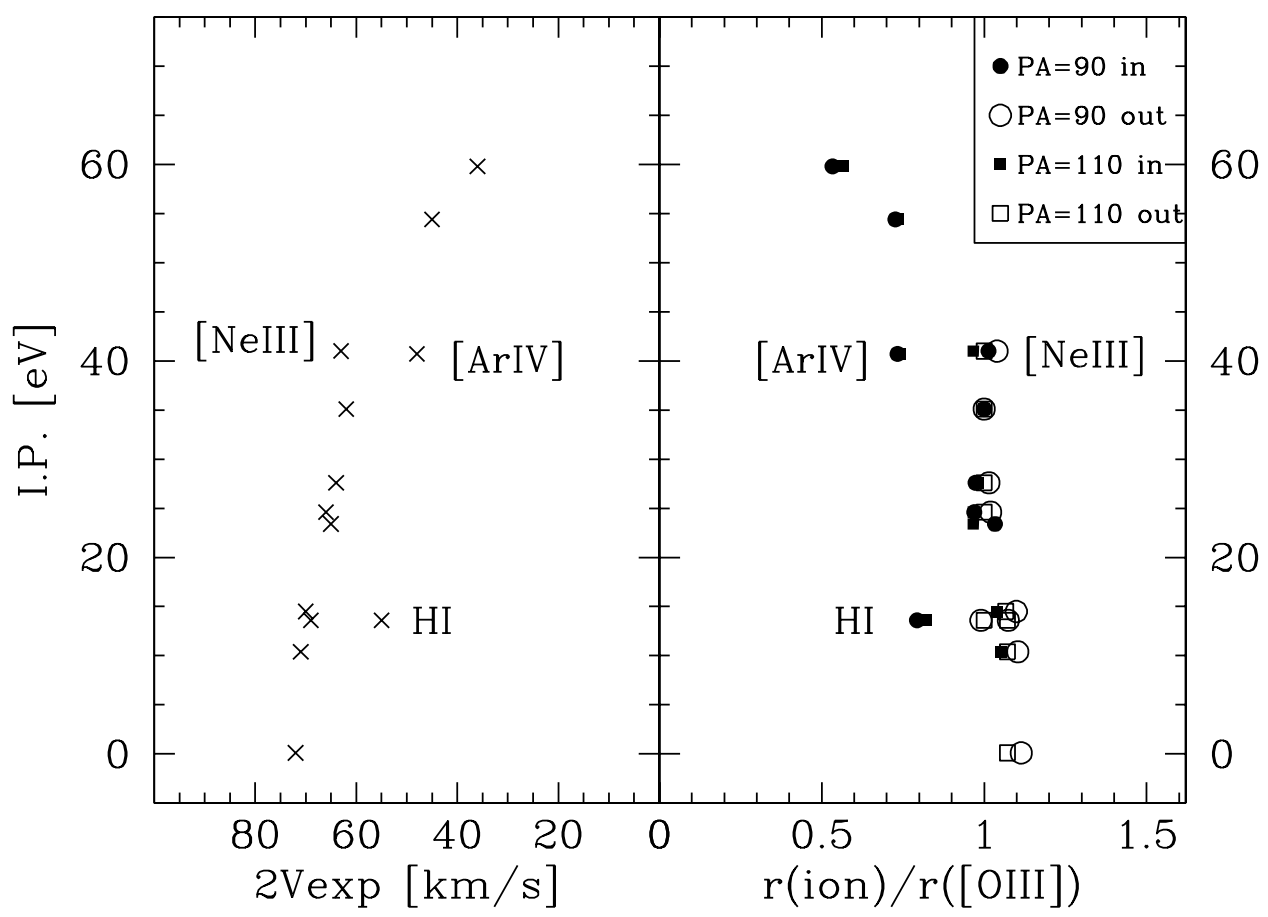

Fig. 3. Expansion velocity in the cspl (left) and peak separation in the zvpc for the inner and the outer shells of NGC 6818 at $\mathrm{PA}=90^{\circ}$ and $110^{\circ}$ (right) vs. IP. Each zvpc peak separation is normalized to the corresponding [O III] value. The ions in peculiar positions are marked.

according to Eq. (1). In other words, they reproduce the tomographic maps in the nebular slices covered by the slit. We have selected He II, [O III] and [N II] as representative of the high, medium and low ionization regions, respectively.

These multicolor $P-V$ maps highlight the large stratification of the radiation and the chaotic nebular structure. NGC 6818 is optically thin in most directions. The low excitation [N II] emission is associated to the presence of some inner and dense layer (see the moustaches, for example). The outermost, faint [N II] spikes detected in PA $=10^{\circ}, 90^{\circ}, 150^{\circ}$ and $170^{\circ}$ belong to the external shell.
The existence of a prominent hole to the North and of a smaller one to the South, as suggested by Rubin et al. (1998), is confirmed. The southern cavity is less evident in Fig. 4, being only grazed by our spectra at $\mathrm{PA}=170^{\circ}$ and $10^{\circ}$. Further hollows are present (like at $\mathrm{PA}=70^{\circ}$ and $90^{\circ}$, in the approaching gas at West of the central star position).

The radial ionization structure of the S-SE edge at PA $=150^{\circ}$, corresponding to the region of the "cometary knot" visible in Fig. 1, is puzzling: [O III] is lacking, whereas He II and $[\mathrm{N} \mathrm{II}]$ are intense in the inner and the outer layers, respectively. 
In summary: although some spectral features of the northern and southern holes can be tentatively interpreted in terms of ionized gas moderately accelerated by some blowing-up agent (as suggested by Rubin et al. 1998), the linear expansion law here adopted represents a valid approximation of the overall nebular kinematics.

\section{The physical conditions}

\subsection{General considerations}

We can obtain the $T$ e radial profile from the diagnostic line ratios of ions in $\mathrm{p}^{2}$ or $\mathrm{p}^{4}$ configurations (like [O III] and [N II]), and the $\mathrm{Ne}$ radial distribution from both the diagnostics of ions in $\mathrm{p}^{3}$ configuration (like [S II]) and the absolute $\mathrm{H} \alpha$ flux. According to Paper IV, the zvpc, which is independent on the expansion velocity field, must be used.

In the specific case of NGC 6818 the large $\mathrm{H} \alpha$ broadening (due to thermal motions, fine structure and expansion velocity gradient) prevents the accurate determination of $F(\mathrm{H} \alpha)_{\text {zvpc }}$ (and then of $\mathrm{Ne}(\mathrm{H} \alpha)$ ). Thus, in this section $T \mathrm{e}[\mathrm{O} \mathrm{III}], T \mathrm{e}[\mathrm{N} \mathrm{II}]$ and $N \mathrm{e}[\mathrm{S} \mathrm{II}]$ are derived from the corresponding line intensity ratios. Later on (Sect. 6.3) we will illustrate the adopted escamotage providing $F(\mathrm{H} \alpha)_{\mathrm{zvpc}}$ and $\mathrm{Ne}(\mathrm{H} \alpha)$ from the observed radial ionization structure and the assumption $\mathrm{O} / \mathrm{H}=$ constant across the nebula.

\subsection{Interstellar absorption}

First of all the observed line intensities must be corrected for interstellar absorption according to:

$\log \frac{I(\lambda)_{\text {corr }}}{I(\lambda)_{\text {obs }}}=f_{\lambda} \mathrm{c}(\mathrm{H} \beta)$

where $f_{\lambda}$ is the interstellar extinction coefficient given by Seaton (1979). The logarithmic extinction at $\mathrm{H} \beta, c(\mathrm{H} \beta)$, is normally obtained by comparing the observed Balmer decrement (in particular $\mathrm{H} \alpha / \mathrm{H} \beta$ ) to the intrinsic value given by Brocklehurst (1971) and Hummer \& Storey (1987). The estimates of $c(\mathrm{H} \beta)$ (from the Balmer ratio) reported in the literature for NGC 6818 span the range 0.25 (Aller \& Czyzak 1983, Liu \& Danziger 1993) to 0.41 (Collins et al. 1961). Moreover, Tylenda et al. (1992) and Condon et al. (1999) obtained $c(H \beta)=0.33$ and 0.40 , respectively, from the radio to $\mathrm{H} \beta$ fluxes.

Thanks to the excellent spatial and spectral accuracies achieved by the superposition technique used $( \pm 0.15$ arcsec and $\pm 1.0 \mathrm{~km} \mathrm{~s}^{-1}$, respectively) we can extend the $\mathrm{H} \alpha / \mathrm{H} \beta$ analysis to the whole spectral image, as recently introduced (Paper IV) in the study of NGC 6565, a compact, dust embedded PN exhibiting a complex $c(\mathrm{H} \beta)$ profile. The results are less dramatic for NGC 6818, since the blurred appearance of both $\mathrm{H} \alpha$ and $\mathrm{H} \beta$ limits the resolution: besides some indications of a soft decline in the innermost regions (likely caused by a local increase of $T \mathrm{e}$ ), the spectral maps appear quite homogeneous at $\mathrm{H} \alpha / \mathrm{H} \beta=3.73( \pm 0.06)$, corresponding to $c(H \beta)=0.37( \pm 0.03)$ (for the case B of Baker \& Menzel 1938, Te $=12000 \mathrm{~K}$ and $\log \mathrm{Ne}=3.00$; Brocklehurst 1971; Aller 1984; Hummer \& Storey 1987).

\section{3. $\mathrm{Te}[\mathrm{O} I I I], \mathrm{Te}[\mathrm{N} I I]$ and $\mathrm{Ne}[\mathrm{S} I I]$}

First we obtain $T \mathrm{e}[\mathrm{O}$ III] from $\lambda 5007 \AA / \lambda 4363 \AA$, the line ratio being almost independent on $N \mathrm{e}$ for $\mathrm{Ne}<10^{4} \mathrm{~cm}^{-3}$. $\mathrm{Ne}$ [S II] is then derived from $\lambda 6717 \AA / \lambda 6731 \AA$ (using $T \mathrm{e}[\mathrm{O}$ III] to take into account the weak dependence of the ratio on $T \mathrm{e}$ ). Last, $T \mathrm{e}[\mathrm{N}$ II] comes from $\lambda 6584 \AA$ $/ \lambda 5755 \AA$ (adopting $N \mathrm{Ne}[\mathrm{S}$ II] for its weak dependence on the electron density).

Although the resulting $T \mathrm{e}[\mathrm{O} \mathrm{III}], T \mathrm{e}[\mathrm{N} \mathrm{II}]$ and $\mathrm{Ne}[\mathrm{S} \mathrm{II}]$ profiles rapidly change with $\mathrm{PA}$, as expected of the chaotic structure of NGC 6818 , nevertheless there are some common features. In order to highlight both the differences and the analogies we have selected two PA close to the apparent major axis $\left(\mathrm{PA}=10^{\circ}\right.$ and $\left.\mathrm{PA}=30^{\circ}\right)$ and two PA close to the apparent minor axis $\left(\mathrm{PA}=90^{\circ}\right.$ and $\left.\mathrm{PA}=110^{\circ}\right)$ as representative of the whole nebular phenomenology.

The results are shown in Fig. 5; their main limitation is evident: due to the weakness of the [N II] auroral line and of the [S II] doublet, $T \mathrm{e}[\mathrm{N} \mathrm{II}]$ and $N \mathrm{e}[\mathrm{S} \mathrm{II}]$ can be obtained only at the corresponding intensity peaks (in the best case).

$T \mathrm{e}[\mathrm{O} \mathrm{III}]$ presents a well-defined radial profile common to all the PA: it is $\geq 15000 \mathrm{~K}$ in the tenuous, innermost regions, it gradually decreases outward down to $12000-12500 \mathrm{~K}$ in the densest layers, and later it remains more or less constant (the last statement is weakened by the auroral line faintness). Such a radial trend is in quantitative agreement with the results by Rubin et al. (1998), based on $\lambda 4363 \AA$ and $\lambda 5007 \AA$ HST/WFPC 2 imagery. The presence of a $T$ e gradient across the nebula is also suggested by Hyung et al. (1999). Previous ground-based $T \mathrm{e}[\mathrm{O} \mathrm{III}]$ determinations are mean values for the brightest (i.e. densest) regions and span the range $11400 \mathrm{~K}$ (de Freitas Pacheco et al. 1991) to $12770 \mathrm{~K}$ (Mathis et al. 1998).

In Fig. $5 T \mathrm{e}[\mathrm{N}$ II] refers to the intensity peaks of the low ionization regions and is systematically below $T \mathrm{e}[\mathrm{O} \mathrm{III}]$, in agreement with both the previous reports for NGC 6818 (9500 K, Hyung et al. 1999, to $11280 \mathrm{~K}$, McKenna et al. 1996) and the general results for PNe (see Aller 1990; Gruenwald \& Viegas 1995; Mathis et al. 1998). More Te values for NGC 6818 are: 11500 K (C III], Kaler 1986), 12700 K and $13800 \mathrm{~K}$ ([Ne V] and [O IV], respectively, Rowlands et al. 1989), 14700 K (Balmer discontinuity, Liu \& Danziger 1993), $12500 \mathrm{~K}$ (C III], Mathis et al. 1998), $13000 \mathrm{~K}$ ([Cl IV], Hyung et al. 1999) and $19000 \mathrm{~K}$ ([O II], Keenan et al. 1999).

In summary, the mean kinetic energy of the free electrons in NGC 6818 is quite large. This on the one hand explains both the $\mathrm{H} \alpha$ broadening and the strength of the forbidden lines (in particular, $\lambda 5007 \AA$ of [O III]), on the other hand is indicative of a very hot central star $\left(T_{*} \geq 150000 \mathrm{~K}\right.$, also supported by the presence of high excitation emissions, up to [Ne V], IP = $97.1 \mathrm{eV}$; see Table 1).

Concerning the [S II] electron densities in the zvpc (Fig. 5), they are limited to the brightest parts of the external, low ionization layers and show peaks up to $2000( \pm 200) \mathrm{cm}^{-3}$. Previously Hyung et al. (1999) obtained $N \mathrm{e}[\mathrm{S} \mathrm{II}] \simeq 2000 \mathrm{~cm}^{-3}$ (they also report $N \mathrm{e}[\mathrm{S} \mathrm{II}] \simeq 3000 \mathrm{~cm}^{-3}$ from the improved calculations for 3 equivalent p-electrons by Keenan et al. 1996). 


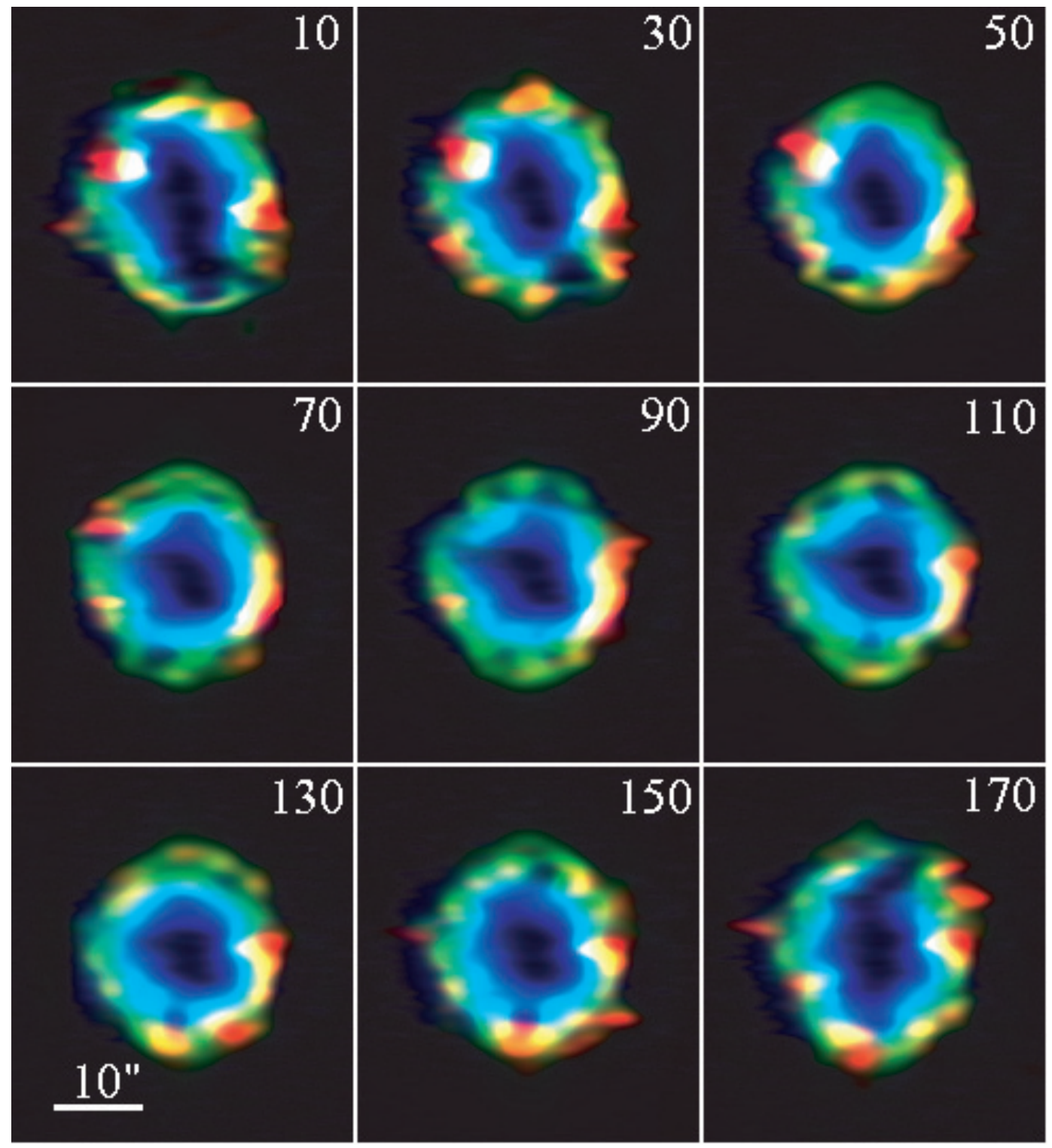

Fig. 4. Position-velocity maps at the nine observed PA for the high (He II, blue), medium ([O III], green) and low ([N II], red) ionization regions of NGC 6818, scaled according to the relation $V \exp \left(\mathrm{km} \mathrm{s}^{-1}\right)=3.5 \times r^{\prime \prime}$. The orientation of these tomographic maps is the same of Fig. 2.

Besides the zvpc, we have extended the $\lambda 6717 \AA / \lambda 6731 \AA$ analysis to the prominent knots of the entire [S II] spectral images. The resulting $\mathrm{Ne}[\mathrm{S} \mathrm{II}]$ values span the range $1500( \pm 200)$ to $2800( \pm 200) \mathrm{cm}^{-3}$, the equatorial moustaches being the densest regions of NGC 6818 .

In the next section we will derive the local filling factor, $\epsilon_{1}$, by combining $N \mathrm{e}[\mathrm{S} \mathrm{II}]$ and $N \mathrm{e}(\mathrm{H} \alpha)_{\text {zvpc }}$, since $N \mathrm{e}[\mathrm{S} \mathrm{II}] \times \epsilon_{1}^{0.5} \simeq$ $\mathrm{Ne}(\mathrm{H} \alpha)_{\text {zvpc }}$ (Aller 1984; Osterbrock 1989).

\section{The radial ionization structure}

\subsection{General considerations}

All the main ionic species are present in the echellograms, with the usual, dreadful handicap for hydrogen: due to the large $\mathrm{H} \alpha$ broadening, the deconvolution for instrumental resolution plus thermal motions plus fine structure appears inadequate, and the detailed $F(\mathrm{H} \alpha)_{\text {zvpc }}$ profile cannot be determined. Moreover:

- the principal $\mathrm{O}^{+}$emissions $(\lambda 3726 \AA$ and $\lambda 3729 \AA)$ fall outside our spectral range. We have considered the much weaker $\lambda 7319.87 \AA$ the strongest line of the red $\mathrm{O}^{+}$quartet;

$-\lambda 3967 \AA$ of [Ne III] being at the extreme blue edge of the frame, the quantitative $\mathrm{Ne}^{++}$analysis appears quite uncertain.

In absence of $\mathrm{H} \alpha$ we are forced to adopt $\lambda 5007 \AA$ as reference line, thus obtaining the radial ionization structure relative to $\mathrm{O}^{++}$according to:

$\frac{\mathrm{X}^{+a}}{\mathrm{O}^{++}}=\frac{F\left(\lambda\left(\mathrm{X}^{+b}\right)\right)_{\mathrm{zvpc}}}{F(\lambda 5007 \AA)_{\mathrm{zvpc}}} f(T \mathrm{e}, N \mathrm{e})$

where $a=b$ for the forbidden lines, and $a=b+1$ for the recombination ones. Note that the solution of the equations of 


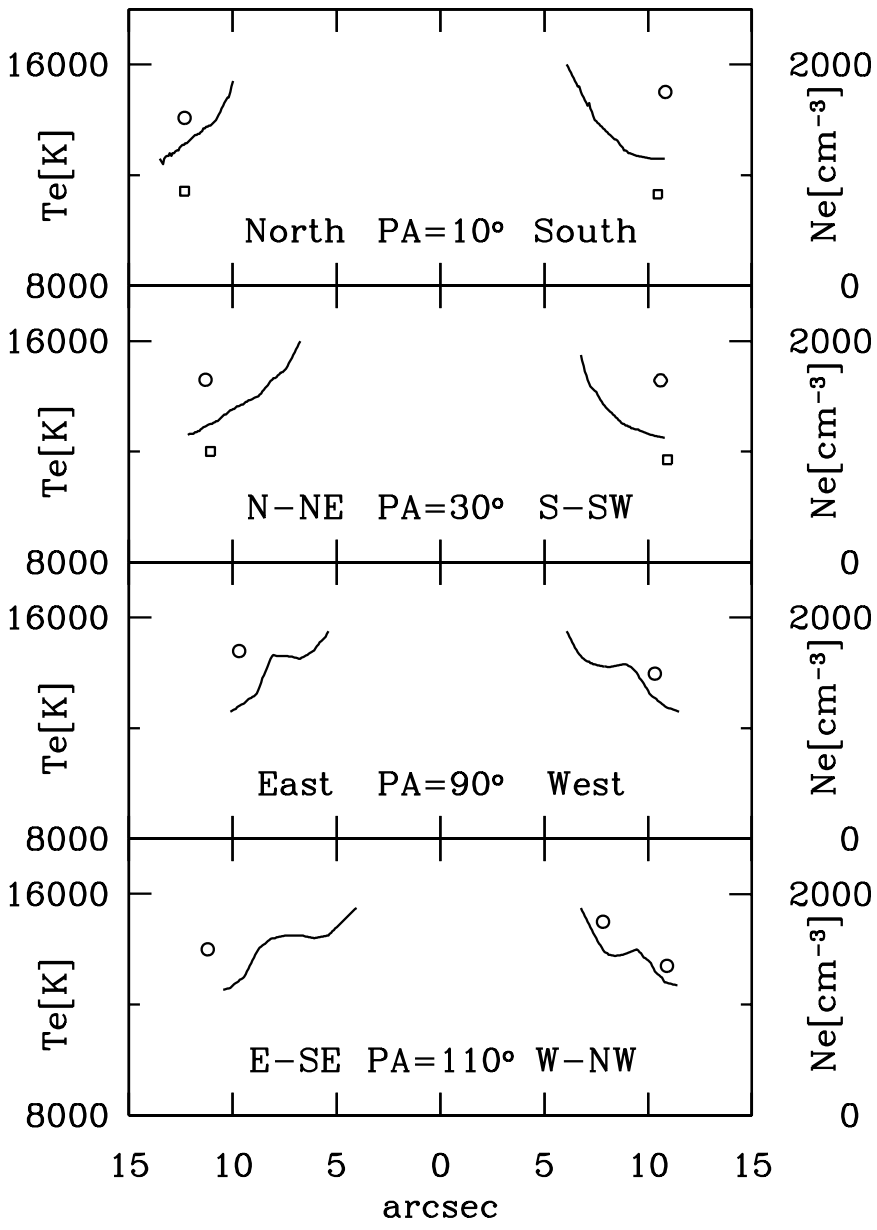

Fig. 5. The diagnostics radial profile at $\mathrm{PA}=10^{\circ}$ and $30^{\circ}$ (close to the major axis of NGC 6818), and at PA $=90^{\circ}$ and $110^{\circ}$ (close to the minor axis). Left ordinate scale: $T \mathrm{e}[\mathrm{O} \mathrm{III}]$ (continuous line) and $T \mathrm{e}[\mathrm{N}$ II] (squares). Right ordinate scale: $N \mathrm{e}[\mathrm{S}$ II] (circles). $T \mathrm{e}[\mathrm{N}$ II] is lacking at $\mathrm{PA}=90^{\circ}$ and $110^{\circ}$, because of the auroral line weakness.

statistical equilibrium requires the detailed knowledge of the plasma diagnostics, whereas $\mathrm{Ne}[\mathrm{S} \mathrm{II}]$ has been derived only at the intensity peaks of the outermost, low ionization layers. Luckily, in the $N \mathrm{e}$ range here considered $f(T \mathrm{e}, N \mathrm{e})$ reduces to $f(T \mathrm{e})$ for most ionic species. Moreover all the emissions, with the exception of $\lambda 5876 \AA$ of $\mathrm{He}$ I and $\lambda 4686 \AA$ of $\mathrm{He}$ II, are forbidden lines, whose emissivity is a direct function of $T \mathrm{e}$. Thus, in first approximation we can put $N \mathrm{e}=1.5 \times 10^{3} \mathrm{~cm}^{-3}$, constant across the nebula; this introduces uncertainties up to $\pm 10 \%$ (for $\mathrm{O}^{+} / \mathrm{O}^{++}$and $\mathrm{Ar}^{+4} / \mathrm{O}^{++}$), which do not modify the results here obtained.

The radial profiles of $\frac{\mathrm{X}^{+i}}{\mathrm{O}^{++}}$at the four selected PA of NGC 6818, shown in Fig. 6, contain a number of interesting features:

- the weakness of the low excitation emissions in PA $=10^{\circ}$ northern sector, $\mathrm{PA}=90^{\circ}$ both sectors and $\mathrm{PA}=110^{\circ}$ both sectors indicates that the nebula is optically thin in these directions. It is almost thick in $\mathrm{PA}=10^{\circ}$ southern sector and PA $=30^{\circ}$ both sectors;

- as expected, $\mathrm{He}^{++} / \mathrm{O}^{++}$decreases outward and $\mathrm{He}^{+} / \mathrm{O}^{++}$increases. They cross for $\mathrm{He}^{++} / \mathrm{O}^{++}=\mathrm{He}^{+} / \mathrm{O}^{++} \simeq 120$, implying that $\mathrm{He}_{\text {tot }} / \mathrm{O}^{++} \simeq 240$. Since in these internal regions
$\mathrm{O}_{\mathrm{tot}} \simeq 1.30 \times \mathrm{O}^{++}$(see Eqs. (10) and (11) in Sect. 6.3) we derive $\mathrm{He} / \mathrm{O} \simeq 185$;

- from similar considerations we infer $\mathrm{N} / \mathrm{O} \simeq 0.23, \mathrm{Ne} / \mathrm{O} \simeq$ $0.15, \mathrm{~S} / \mathrm{O} \simeq 0.012$ and $\mathrm{Ar} / \mathrm{O} \simeq 0.008$.

These chemical abundances (relative to oxygen) must be compared with the corresponding values (relative to hydrogen) obtained from the conventional method, as illustrated in the next Section.

\subsection{Total chemical abundances}

According to the critical analysis by Alexander \& Balick (1997) we consider the total line fluxes (i.e. integrated over the whole spatial profile and the expansion velocity field). The resulting ionic abundances must be multiplied for the corresponding ICFs, the correcting factors for the unobserved ionic stages. These were obtained both empirically (Barker 1983, 1986) and from interpolation of theoretical nebular models (Shields et al. 1981; Aller \& Czyzak 1983; Aller 1984; Osterbrock 1989).

The final mean chemical abundances of NGC 6818, presented in Table 3 (last column), are in reasonable agreement with the previous estimates reported in the literature (also listed in the Table), and in excellent agreement with the indications of Sect. 6.1 .

\section{3. $\mathrm{F}(\mathrm{H} \alpha)_{z v p c}$ and $\mathrm{Ne}(\mathrm{H} \alpha)$}

As emphasized in Paper IV, the $\mathrm{H} \alpha$ flux distribution in the zvpc, $F(\mathrm{H} \alpha)_{\mathrm{zvpc}}$, is the fundamental parameter linking $N \mathrm{e}$ with both the spatial and the kinematical properties of the expanding plasma through the relation:

$N \mathrm{e}=\frac{1.19 \times 10^{9}}{T \mathrm{e}^{-0.47}} \times\left(\frac{F(\mathrm{H} \alpha)_{\text {zvpc }}}{\epsilon_{1} \times r_{\text {cspl }} \times D}\right)^{1 / 2}$

where:

$-D$ is the nebular distance;

$-r_{\text {cspl }}$ is the angular radius of the cspl (i.e. the nebular size in the radial direction);

$-\epsilon_{1}$ is the "local filling factor", representing the fraction of the local volume actually filled by matter with density $\mathrm{Ne}$.

In order to recover $F(\mathrm{H} \alpha)_{\text {zvpc }}$ we start assuming $\mathrm{O} / \mathrm{H}=5.5 \times$ $10^{-4}$, constant across the nebula. At each radial position:

$\frac{\mathrm{O}}{\mathrm{H}}=\frac{\sum_{i=0}^{8} \mathrm{O}^{0+i}}{\mathrm{H}^{0}+\mathrm{H}^{+}}$

It can be written in the form:

$\frac{\mathrm{O}}{\mathrm{H}}=\frac{\mathrm{O}^{++}}{\mathrm{H}^{+}} \times i c f\left(\mathrm{O}^{++}\right)$.

That is:

$\frac{\mathrm{O}}{\mathrm{H}}=\frac{F(\lambda 5007 \AA)_{\mathrm{zvpc}}}{F(\mathrm{H} \alpha)_{\mathrm{zvpc}}} \times f(T \mathrm{e}, N \mathrm{e}) \times i c f\left(\mathrm{O}^{++}\right)$.

Thus obtaining:

$F(\mathrm{H} \alpha)_{\mathrm{zvpc}}=$

$1.9 \times 10^{3} F(\lambda 5007 \AA)_{z v p c} \times f(T \mathrm{e}, N \mathrm{e}) \times i c f\left(\mathrm{O}^{++}\right)$. 


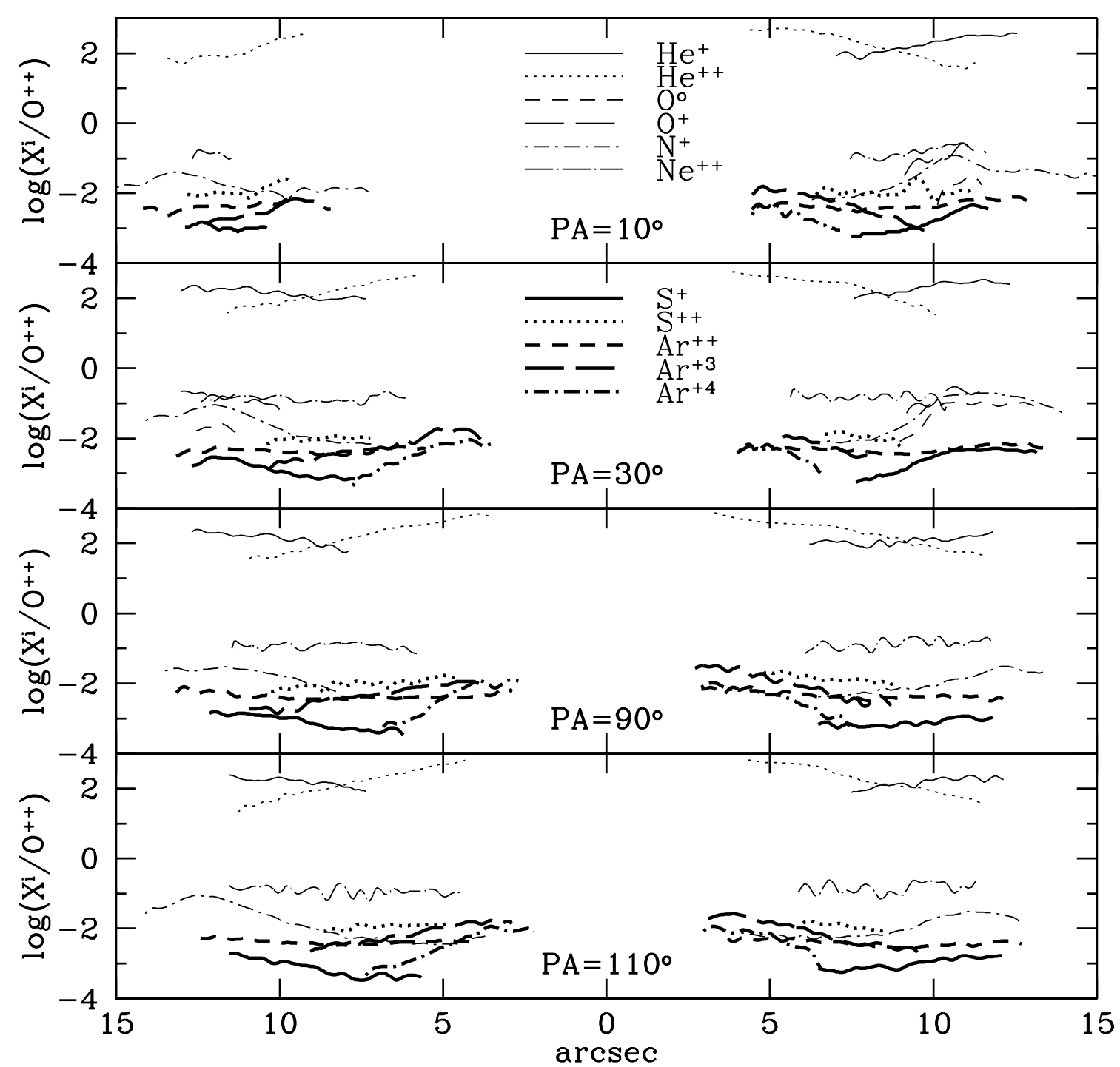

Fig. 6. The radial ionization structure (relative to $\mathrm{O}^{++}$) at $\mathrm{PA}=10^{\circ}$ and $30^{\circ}$ (close to the major axis of NGC 6818 ), and at $\mathrm{PA}=90^{\circ}$ and $110^{\circ}$ (close to the minor axis). The orientation is as in Fig. 5.

Also in this case $f(T \mathrm{e}, \mathrm{Ne})$ essentially reduces to $f(T \mathrm{e})$, with opposite trends for the emissivity of the forbidden and the recombination line.

Equation (8) provides the $\mathrm{H} \alpha$ flux distribution in the zvpc (and $\mathrm{Ne}(\mathrm{H} \alpha)$ through Eq. (4)) once the ionization correcting factor ic $f\left(\mathrm{O}^{++}\right)$is known. The complex structure of NGC 6818 implies that ic $f\left(\mathrm{O}^{++}\right)$strongly changes across the nebula. In the innermost regions we have: $\mathrm{O}^{0} / \mathrm{O}^{++}<<1, \mathrm{O}^{+} / \mathrm{O}^{++}<<1$ and $\mathrm{H}^{0} / \mathrm{H}^{+}<<1$. Thus icf $\left(\mathrm{O}^{++}\right)$becomes:

$i c f\left(\mathrm{O}^{++}\right)_{\mathrm{inner}}=1+\frac{\sum_{i=3}^{8} \mathrm{O}^{+i}}{\mathrm{O}^{++}}$

According to Seaton (1968), $\frac{\sum_{i=3}^{8} \mathrm{O}^{+i}}{\mathrm{O}^{++}}$can be derived from the ionization structure of helium thanks to the closeness of the $\mathrm{O}^{++}$and $\mathrm{He}^{+}$ionization potentials (54.9 and $54.4 \mathrm{eV}$, respectively). To this end we have performed a number of photo-ionization simulations (see also Alexander \& Balick 1997) obtaining the fairly good relation:

$\frac{\sum_{i=3}^{8} \mathrm{O}^{+i}}{\mathrm{O}^{++}} \simeq 0.30 \times \frac{\mathrm{He}^{++}}{\mathrm{He}^{+}}$

Therefore we will adopt:

icf $\left(\mathrm{O}^{++}\right)_{\text {inner }}=1+0.30 \times \frac{\mathrm{He}^{++}}{\mathrm{He}^{+}}$.

Concerning the outermost nebula, the contribution of $\sum_{i=3}^{8} \mathrm{O}^{+i}$ can be neglected. Moreover in these regions we have $\mathrm{O}^{0} / \mathrm{O}^{+}<1$ (see Fig. 6), implying a low efficiency of the chargeexchange reaction $\mathrm{O}^{+}+\mathrm{H}^{0} \leftrightarrows \mathrm{O}^{0}+\mathrm{H}^{+}$(Williams 1973; Aller 1984; Osterbrock 1989), i.e. $\mathrm{H}^{0}<<\mathrm{H}^{+}$. Thus:

$i c f\left(\mathrm{O}^{++}\right)_{\text {outer }}=1+\frac{\mathrm{O}^{0}}{\mathrm{O}^{++}}+\frac{\mathrm{O}^{+}}{\mathrm{O}^{++}}$.

All this is summarized in Fig. 7, showing the $\mathrm{Ne}$ radial profile (at the four selected PA of NGC 6818) obtained from $F(\mathrm{H} \alpha)_{\mathrm{zvpc}}$ 
Table 3. Total chemical abundances (relative to hydrogen).

\begin{tabular}{lccccc}
\hline \hline Element & $\begin{array}{c}\text { Aller \& Czyzak } \\
(1983)\end{array}$ & $\begin{array}{c}\text { de Freitas Pacheco et al. } \\
(1991)\end{array}$ & $\begin{array}{c}\text { Liu \& Danziger } \\
(1993)\end{array}$ & $\begin{array}{c}\text { Hyung et al. } \\
(1999)\end{array}$ & This paper \\
\hline $\mathrm{He}$ & 0.107 & 0.126 & 0.114 & 0.105 & $0.106( \pm 0.003)$ \\
$\mathrm{C}$ & $4.47 \times 10^{-4}$ & - & - & $8.0 \times 10^{-4}$ & - \\
$\mathrm{N}$ & $1.41 \times 10^{-4}$ & $1.1 \times 10^{-4}$ & - & $4.0 \times 10^{-4}$ & $1.4( \pm 0.2) \times 10^{-4}$ \\
$\mathrm{O}$ & $5.50 \times 10^{-4}$ & $5.25 \times 10^{-4}$ & $6.37 \times 10^{-4}$ & $7.0 \times 10^{-4}$ & $5.5( \pm 0.5) \times 10^{-4}$ \\
$\mathrm{Ne}$ & $1.23 \times 10^{-4}$ & - & - & $1.0 \times 10^{-4}$ & $9.0( \pm 2.0) \times 10^{-5}$ \\
$\mathrm{Na}$ & $3.09 \times 10^{-6}$ & - & - & $3.0 \times 10^{-6}$ & - \\
$\mathrm{Mg}$ & - & - & - & $3.0 \times 10^{-5}$ & - \\
$\mathrm{Si}$ & - & - & - & $9.0 \times 10^{-6}$ & - \\
$\mathrm{S}$ & $8.9 \times 10^{-6}$ & $1.2 \times 10^{-5}$ & - & $7.0 \times 10^{-6}$ & $6.2( \pm 1.4) \times 10^{-6}$ \\
$\mathrm{Cl}$ & $1.9 \times 10^{-7}$ & - & - & $3.0 \times 10^{-7}$ & - \\
$\mathrm{Ar}$ & $3.8 \times 10^{-6}$ & - & - & $4.0 \times 10^{-6}$ & $4.0( \pm 0.8) \times 10^{-6}$ \\
$\mathrm{~K}$ & $1.02 \times 10^{-7}$ & - & - & $2.0 \times 10^{-7}$ & - \\
$\mathrm{Ca}$ & $1.15 \times 10^{-7}$ & - & - & $1.5 \times 10^{-7}$ & - \\
\hline
\end{tabular}

for some representative values of $\epsilon_{1} \times r_{\mathrm{cspl}} \times D$ (in arcsec kpc), superimposed to $\mathrm{Ne}[\mathrm{S} \mathrm{II}]$ (taken from Fig. 5).

Figure 7 evidences the basic advantage of using $F(\mathrm{H} \alpha)_{\mathrm{zvpc}}$ in the determination of the electron density radial distribution: $\mathrm{Ne}(\mathrm{H} \alpha)$ extends all over the nebular image, whereas $\mathrm{Ne}[\mathrm{S}$ II] is limited to the peaks of the low excitation regions.

In detail:

- at $\mathrm{PA}=10^{\circ}$ (along the apparent major axis), $N \mathrm{e}(\mathrm{H} \alpha)$ presents a single, broad and asymmetric (i.e. steeper outwards) bell-shaped profile;

- the double-peak structure is very subtle at $\mathrm{PA}=30^{\circ}$ (close to the apparent major axis), the inner peaks being predominant; - at both $\mathrm{PA}=90^{\circ}$ and $110^{\circ}$ (close to the apparent minor axis) the two-shell distribution clearly appears; the $\mathrm{Ne}(\mathrm{H} \alpha)$ top corresponds to the inner peaks at $\mathrm{PA}=110^{\circ}$, whereas at $\mathrm{PA}=90^{\circ}$ the peaks are almost equivalent;

- an outward, low density tail is present at all directions, extending up to about 15 arcsec from the star.

Note in Fig. 7 the match between $\mathrm{Ne}(\mathrm{H} \alpha)$ and $N \mathrm{e}[\mathrm{S} \mathrm{II}]$ for $\epsilon_{1} \times r_{\text {cspl }} \times D \simeq 9.5( \pm 1)$ arcsec kpc, implying that $\epsilon_{1} \times D \simeq$ $0.88( \pm 0.10) \mathrm{kpc}$ (see Table 2 and Sect. 4). Although the assumption $\epsilon_{1}=1$ provides a lower limit of $0.9 \mathrm{kpc}$ for the nebular distance, we can no longer delay a better quantification of this fundamental parameter.

\section{The nebular distance, mass and age}

The only individual distance reported in the literature (based on rough parameters for the star and the nebula) goes back to Gurzadyan (1970), who derived an indicative value of $2.2 \mathrm{kpc}$ from the observed to expected size of the $\mathrm{He}^{++}$zone.

As compensation the nebula is present in a good 20 catalogues of statistical distances (listed in Table 4). They provide the following mean values:

$<D>($ Shklovsky $) \simeq 2.0( \pm 0.4) \mathrm{kpc}$;

$<D>$ (ionized mass-radius relation $) \simeq 1.7( \pm 0.4) \mathrm{kpc}$;

$<D>$ (surface brightness-radius relation $) \simeq 1.3( \pm 0.4) \mathrm{kpc}$;

$<D>$ (other methods $) \simeq 2.8( \pm 1.0) \mathrm{kpc}$.
To be noticed that the overall properties inferred in the previous sections (in synthesis: NGC 6818 is an optically thin, almost thin in some directions, quite "normal" PN) suggest that $<D>$ (Shklovsky),$<D>$ (ionized mass-radius relation) and $<D>$ (surface brightness-radius relation) are, at least grossly, reliable.

In order to derive the dynamical parallax we have analysed the first and second epoch (1998.30 and 2000.45, respectively) HST images of NGC 6818, searching for the angular expansion of the ionized gas; when combined with Eq. (1), it provides a reliable nebular distance (Reed et al. 1999; Palen et al. 2002). Since the target is at the centre of the planetary camera (PC) chip in the 2000.45 images, whereas it is quite off-axis in the 1998.30 ones, the correction for optical camera distortions was performed with the IRAF/STSDAS task "drizzle" (see Fruchter $\&$ Hook 2002) using the Trauger coefficients.

No apparent shift is obtained from the couples of [O III] and [N II] frames.

According to Reed et al. (1999), we infer that the angular expansion of the gas is $<1.2 \times 10^{-2} \operatorname{arcsec}$, that is $\frac{\mathrm{d} \theta}{\mathrm{d} t}<$ $5.5 \times 10^{-3} \operatorname{arcsec} \mathrm{yr}^{-1}$. Since $D(\mathrm{pc})=0.211\left[V \exp \left(\mathrm{km} \mathrm{s}^{-1}\right) / \frac{\mathrm{d} \theta}{\mathrm{d} t}\right.$ $\left.\left(\operatorname{arcsec} \mathrm{yr}^{-1}\right)\right]$, we derive $D(\mathrm{NGC} 6818)>1300 \mathrm{pc}$.

We have attempted a better quantification of $D$ (NGC 6818) through the interstellar absorption-distance relation (Lutz 1973; Gathier et al. 1986; Saurer 1995) given by the field stars with accurate $m_{V}, m_{B}$, spectral type and luminosity class. They were selected using the SIMBAD facilities of the CDS, Strasbourg Astronomical Observatory.

The resulting $A_{\mathrm{V}}$-distance law is presented in Fig. 8, where the small cluster of data at the right edge refers to the supergiant stars of NGC 6822 (Barnard's galaxy), a nearby Ir galaxy projected at about 40 arcmin S-SE of our nebula.

Figure 8 indicates that:

- the galactic absorption rapidly changes in direction (note the spread of the low-weight points). This is confirmed by the inspection of both the ESO/SERC and the Palomar Schmidt surveys, showing a variable background crossed by a series of extended, broad and faint emitting filaments;

- close to the nebula direction (high-weight data), $A_{\mathrm{V}}$ quickly increases up to $D \simeq 1.0-1.5 \mathrm{kpc}$, and later is 


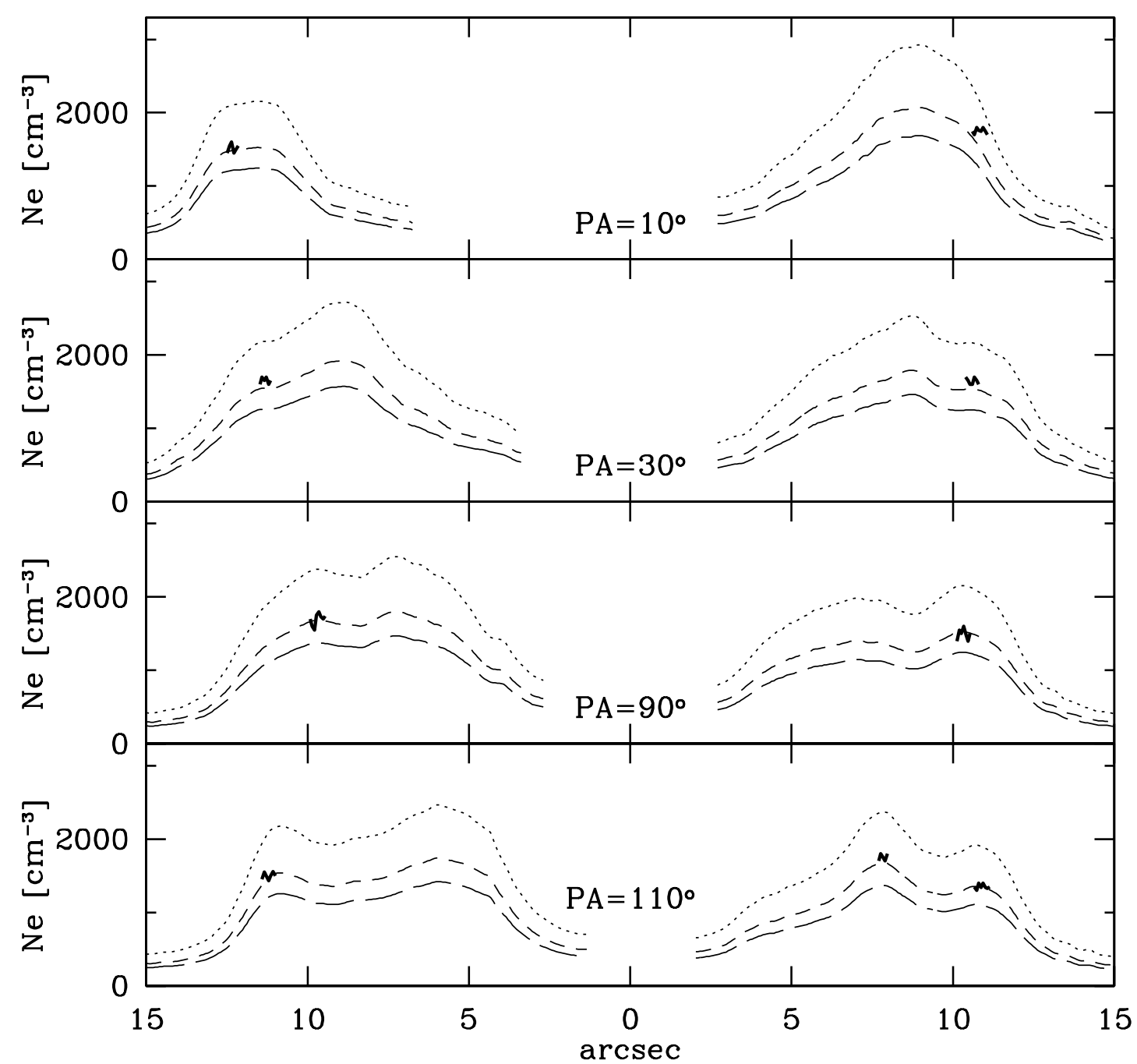

Fig. 7. The $N \mathrm{e}(\mathrm{H} \alpha)_{\text {zvpc }}$ radial profile at the four selected PA of NGC 6818 for some representative values of $\epsilon_{1} \times r_{\text {cspl }} \times D$ (dotted line $=5$ arcsec kpc, short-dashed line $=10$ arcsec kpc, long-dashed line $=15$ arcsec kpc), superimposed to $N \mathrm{e}$ [S II] (thick line; same as Fig. 5). The orientation is as in Figs. 5 and 6.

constant (as expected of the large galactic latitude of the field, $|b I I| \simeq 18^{\circ}$ ). Note that for the super-giant stars of the nearby galaxy NGC 6822 we have $A_{\mathrm{V}}$ (tot) $=$ $A_{\mathrm{V}}($ galactic $)+A_{\mathrm{V}}(\mathrm{NGC} 6822)$, where $A_{\mathrm{V}}(\mathrm{NGC} 6822)$ changes from star to star, and $A_{\mathrm{V}}($ galactic $)=$ const. For $A_{\mathrm{V}}(\mathrm{NGC} 6822)=0$ we infer $A_{\mathrm{V}}($ galactic $) \simeq 0.80( \pm 0.1)$, in excellent agreement with the literature reports (see Gallart et al. 1996; Bianchi et al. 2001);

- from $A_{\mathrm{V}}=2.18 \times c(\mathrm{H} \beta)($ Acker 1978) we have $A_{\mathrm{V}}(\mathrm{NGC} 6818)=0.81( \pm 0.06)$ and $D(\mathrm{NGC} 6818)$ $\geq 1.5 \mathrm{kpc}$.

Although in Fig. 8 all the solutions for $D(\mathrm{NGC} \mathrm{6818)} \geq 1.5 \mathrm{kpc}$ appear legitimate, both the large galactic latitude of the nebula $\left(|b I I| \simeq 18^{\circ}\right)$ and the low radial velocity relative to the Local Standard of Rest, $V_{\mathrm{LSR}}=-1.7 \mathrm{~km} \mathrm{~s}^{-1}$ (Sect. 4), decidedly favour the lowest values, i.e. $1.5 \mathrm{kpc} \leq D(\mathrm{NGC} 6818) \leq 2.0 \mathrm{kpc}$ (in agreement with the information previously obtained from the statistical distance scales).
In the following we will adopt $D(\mathrm{NGC}$ 6818) = $1.7\left(\begin{array}{c}+0.3 \\ -0.2\end{array}\right) \mathrm{kpc}$, corresponding to a distance from the galactic plane $|z| \simeq 0.5 \mathrm{kpc}$.

The local filling factor in the nebula is $\epsilon_{1} \simeq 0.5$. The ionized mass (obtained in different ways: from the $\mathrm{H} \beta$ flux, the radio flux and the observed Ne distribution; Aller 1984; Pottasch 1984; Osterbrock 1989 and Paper IV) results to be $M_{\text {ion }} \simeq$ $0.13( \pm 0.03) M_{\odot}$, and the kinematical age $t_{\text {kin }} \propto r / V \exp \simeq$ $2300( \pm 300) \mathrm{yr}$.

$M_{\text {ion }}$ is close to the total nebular mass, NGC 6818 being an optically thin (almost thin in some directions) PN, whereas $t_{\text {kin }}$ represents a lower limit to the actual age, $t_{\mathrm{NGC}} 6818$, since the dynamical history of the gas is unknown. We can obtain a reliable estimate of $t_{\mathrm{NGC}} 6818$ by assuming a nebular ejection at $V \exp _{\text {superwind }}<V \exp _{\mathrm{NGC}} 6818$, followed by a constant acceleration up to $V \exp _{\mathrm{NGC}} 6818$. $V \exp _{\text {superwind }}$ comes from the $\mathrm{OH} / \mathrm{IR}$ sources, commonly regarded as the PNe precursors (Habing 1996). For $V \exp _{\text {superwind }}=15( \pm 5) \mathrm{km} \mathrm{s}^{-1}$ (Chengalur et al. 1993; David et al. 1993; Sjouwerman et al. 1998) we derive $t_{\mathrm{NGC} 6818}=3500( \pm 400) \mathrm{yr}$, i.e. our nebula is rather young. 
Table 4. Statistical distances of NGC 6818.

\begin{tabular}{lll}
\hline \hline Author & Distance (kpc) & Method \\
\hline O’Dell (1962) & 1.7 & Shklovsky (1956), i.e. ionized mass = const. \\
Cahn \& Kaler (1971) & $1.82-2.38$ & Shklovsky \\
Cudworth (1974) & 1.95 & proper motions \\
Milne \& Aller (1975) & 2.07 & Shklovsky (radio) \\
Cahn (1976) & 2.2 & Shklovsky \\
Acker (1978) & 1.6 & published statistical distance scales re-calibrated with individual distances \\
Maciel \& Pottasch (1980) & 1.46 & ionized mass-radius relation \\
Daub (1982) & 1.29 & ionized mass-radio surface brightness relation \\
Phillips \& Pottasch (1984) & 4.45 & observed vs. predicted radio fluxes \\
Maciel (1984) & 1.5 & ionized mass-radius relation \\
Amnuel et al. (1984) & 0.95 & radio surface brightness-radius relation \\
Kingsburgh et al. (1992) & 2.46 & Shklovsky \\
Cahn et al. (1992) & 1.87 & ionized mass-surface brightness relation \\
van de Steene \& Zijlstra (1995) & 1.58 & radio continuum surface brightness temperature-radius relation \\
Zhang (1995) & 1.68 & ionized mass-radius and radio cont. surface bright. temp.-radius relations \\
Gorny et al. (1997) & 2.1 & theoretical evolutionary tracks vs. observed nebular and stellar parameters \\
Mal'kov (1997) & 2.5 & theoretical evolutionary age vs. observed dynamical age \\
Cazetta \& Maciel (2000) & $>2.9$ & Peimbert \& Torres-Peimbert (1983) class $\leftrightarrows$ stellar mass $\leftrightarrows$ stellar gravity and \\
& & temperature $\leftrightarrows$ stellar luminosity \\
Bensby \& Lundström (2001) & 2.23 & ionized mass-radius relation \\
Phillips (2002) & 0.63 & radio surface brightness-radius relation \\
\hline
\end{tabular}

\section{The central star}

As mentioned in Sect. 2, the exciting star of NGC 6818 is the northern component of a visual binary system. The WFPC2 frames taken through the broad-band filter $\quad$ F555W $\quad\left(\lambda_{\mathrm{c}}=5407 \AA\right.$, bandwidth $\left.=1236 \AA\right) \quad$ give $m_{V}=17.06( \pm 0.05)$ for the central star and $m_{V}=17.73( \pm 0.05)$ for the southern red companion, where the unknown star colors are the main source of inaccuracy. Please note that for $D=1.7 \mathrm{kpc}$ we have $\left(M_{V}\right)_{0}($ red companion $) \simeq+5.75$, as expected of a late spectral type (G8 to K0) Main Sequence star: a further, although weak, sign in favour of the adopted distance.

According to Tylenda et al. (1993) and Feibelman (1994), the exciting star of NGC 6818 presents a weak emission line spectrum in both the UV and the optical regions. In order to derive the $\mathrm{H}$ I and $\mathrm{He}$ II Zanstra temperatures, $T_{\mathrm{Z}} \mathrm{H}$ I and $T_{\mathrm{Z}} \mathrm{He}$ II respectively, we have obtained the total $\mathrm{H} \beta$ and $\lambda 4686 \AA$ nebular fluxes from the overall line profile at each PA, assuming a circular symmetry of the spectral image. We find log $F(\mathrm{H} \beta)_{\text {obs }}=-10.49( \pm 0.03) \mathrm{mW} \mathrm{m}^{-2}$ and $F(\lambda 4686 \AA) / F(\mathrm{H} \beta)=$ $0.65( \pm 0.04)$, in excellent agreement with the values reported by Aller \& Czyzak (1979), Kohoutek \& Martin (1981), Webster (1983), Acker et al. (1991), and Hyung et al. (1999).

The resulting Zanstra temperatures are $\log \left(T_{\mathrm{Z}} \mathrm{H} \mathrm{I}\right)=$ $5.20( \pm 0.04)$ and $\log \left(T_{\mathrm{Z}} \mathrm{He}\right.$ II $)=5.24( \pm 0.04)$. We note in passing that $T_{\mathrm{Z}} \mathrm{HI} \simeq T_{\mathrm{Z}} \mathrm{He} \mathrm{II}$; this is quite surprising for a high excitation, optically thin (almost thin in some directions) PN. Previous determinations (Harman \& Seaton 1966; Martin 1981; Gathier \& Pottasch 1988; Mal'kov 1997) span the range 4.82 to 5.20 for $\log \left(T_{\mathrm{Z}} \mathrm{H} \mathrm{I}\right)$, and 5.03 to 5.30 for $\log \left(T_{\mathrm{Z}} \mathrm{He}\right.$ II). Moreover Pottasch \& Preite-Martinez (1983) and Preite-Martinez et al. (1991) find $\log T_{*}=4.93$ and 5.02-5.05, respectively, from the energy balance of the nebula.
The stellar luminosities are $\log L_{*} / L_{\odot}\left(T_{\mathrm{Z}} \mathrm{H} \mathrm{I}\right)=3.0( \pm 0.1)$ and $\log L_{*} / L_{\odot}\left(T_{\mathrm{Z}} \mathrm{He}\right.$ II $)=3.1( \pm 0.1)$ (using the bolometric corrections by Schönberner 1981, and adopting $\mathrm{D}=1.7 \mathrm{kpc}$ ).

NGC 6818 results to be a rather young PN surrounding a very hot star of relatively low luminosity. This suggests that: - the central star is a hydrogen-burning post-AGB star (the helium-burning ones evolve too slowly);

- the stellar mass, $M_{*}$, is larger than the average value $\left(\simeq 0.60 M_{\odot}\right)$ of the PNe nuclei (Blöcker 1995 and references therein).

The detailed comparison with the theoretical evolutionary tracks by Schönberner (1981, 1983), Iben (1984), Wood \& Faulkner (1986), Blöcker \& Schönberner (1990), Vassiliadis \& Wood (1994) and Blöcker (1995) confirms that no solutions are possible for the He-burning post-AGB stars (they are too slow), whereas the H-burning nuclei give $M_{*}=0.625-0.65 M_{\odot}$.

Moreover, the position of the central star of NGC 6818 in the $\log L-\log T$ diagram coincides with the beginning of the luminosity decline at the end of the shell nuclear burning. In this evolutionary phase all models present a large luminosity gradient with a deep minimum at $\log L_{*} / L_{\odot} \simeq 3.0$, i.e. the value obtained for the central star of NGC 6818. For example the $0.625 M_{\odot}$ H-burning nucleus by Blöcker (1995) (showing an astonishing resemblance with our star) has $\log L_{*} / L_{\odot}=3.387$ at $t=3457 \mathrm{yr}$, and $\log L_{*} / L_{\odot}=2.846$ at $t=3585 \mathrm{yr}$, i.e. it drops in luminosity by a factor of 3.5 in $128 \mathrm{yr}$.

And indeed, the central star of NGC 6818 is clearly visible in the earliest photographic reproduction of the nebula (Pease 1917; plate taken on July 12, 1912 with the Mount Wilson 60 -inch reflector); the rough photometric sequence defined by the field stars gives $m_{B}=15.2( \pm 0.4)$. More stellar magnitude estimates reported in the literature are: $m_{\mathrm{pg}}=14$ (Curtis 1918), $m_{\mathrm{pg}}=15$ (Berman 1937), $m_{\mathrm{pv}}=15.6$ (Cudworth 1974), $m_{V} \simeq 14.9$ (Martin 1981), $m_{B}>15$ (Shaw \& Kaler 1985), 


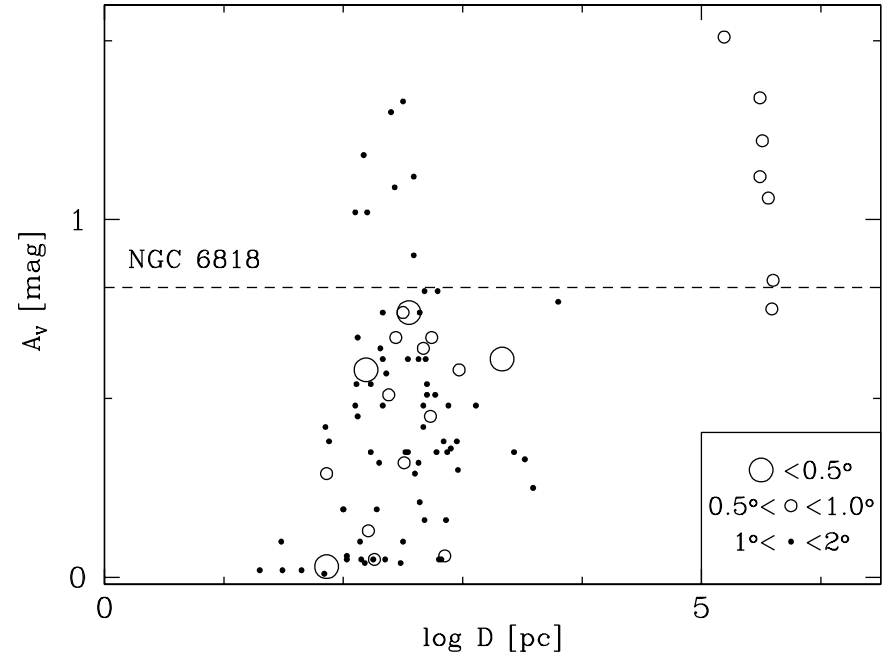

Fig. 8. The interstellar absorption-distance relation for the field stars of NGC 6818. Three symbols are used, referring to stars at different angular separation from the nebula.

$m_{B}=16.97$ and $m_{V}=17.02$ (Gathier \& Pottasch 1988), and $m_{V}=17.06$ (this paper, referring to the epoch 1998-2000).

In addition, the first spectroscopic observation of NGC 6818 (star+nebula, Palmer 1903, referring to 1901) indicates "a pretty strong continuum spectrum extending out to $\lambda 372$, with the following bright lines: $\mathrm{H} \beta, \mathrm{H} \gamma, \mathrm{H} \delta \ldots .$. . Palmer observed a large sample of nebulae with the Crossley reflector+plate spectrograph, reporting that "the faintest continuum spectrum photographed was that of a fifteenth magnitude star".

In spite of the photometric heterogeneity of these data, the suspicion of a "historical" decline for the nucleus of NGC 6818 appears legitimate (and robust). Also note that the magnitudes of the star just before the luminosity drop were: $m_{V} \simeq m_{\mathrm{pv}} \simeq$ 14.5 and $m_{B} \simeq m_{\mathrm{pg}} \simeq 14.0$ (for $D=1.7 \mathrm{kpc}$ and $c(\mathrm{H} \beta)=0.37$ ).

In summary, all the evidence is that:

- the quite massive central star is rapidly fading,

- the thin-thick transition is hanging over the nebula.

Within this scenario the following caveat is in order: because of the luminosity drop of the fast evolving star, the ionization and thermal structure of NGC 6818 are out of equilibrium. The recombination processes prevail, being faster in the high ionized species (Tylenda 1986; Marten \& Szczerba 1997); this qualitatively explains the observed, unexpected closeness of the $\mathrm{H}$ I and He II Zanstra temperatures: the nebula is still optically thin (almost thin in some directions), but $\mathrm{H} \beta$ maintains a "longer memory" than $\lambda 4686 \AA$ of the past stellar luminosity. Although the large $\mathrm{Ne}$ of the gas implies a short "time lag" of the main nebula (a few dozen years), nevertheless both $T_{\mathrm{Z}} \mathrm{He}$ II and, even more, $T_{\mathrm{Z}} \mathrm{H}$ I are slightly over-valued. In other words: NGC 6818 is over-luminous with respect to the present UV stellar flux.

The overall picture of our nebula shows a remarkable series of analogies with NGC 6565. According to Paper IV, NGC 6565 is a young (2300 yr), optically thick ellipsoid embedded in a large cocoon of neutral, dusty gas. It is in a deep recombination phase (started about $400 \mathrm{yr}$ ago), caused by the luminosity drop of the massive powering $\operatorname{star}\left(M_{*} \simeq 0.65 M_{\odot}\right)$, which has almost reached the white dwarf locus $\left(\log L_{*} / L_{\odot} \simeq\right.$ $2.0, \log T_{*} \simeq 5.08$ ).

All this suggests the evolutive contiguity of the two PNe, NGC 6818 being a bit older, but also a bit less "mature" than NGC 6565 , i.e. the central star of the latter is more massive. No doubt NGC 6818 will develop in the near future most of the observational characteristics already present in NGC 6565 (and in the other recombining PNe contained in Tylenda 1986 and Corradi et al. 2000).

We have also carried out a search for other PNe in the peculiar evolutionary phase of NGC 6818 (e.g. at the very beginning of the thin-thick transition) adopting the following criteria: high temperature and quite low luminosity of the star, different [O III] and [N II] nebular morphology, high excitation class and relatively weak low ionization emissions. Among the dozen or so candidates we identified, the most promising are NGC 6326, NGC 6884, NGC 7354 and IC 4663.

\section{The photo-ionization model}

\subsection{General approach}

In this section the photo-ionization code CLOUDY (Ferland et al. 1998) is applied to a PN having the same distance, matter distribution and chemical composition of NGC 6818.

We immediately point out that CLOUDY is a "steady-state" model (a constant UV flux hits the nebula), whereas we argued that the ionization and thermal structure of NGC 6818 are out of equilibrium, because of the fast luminosity drop of the star. A similar, but less dramatic situation occurs in the recombining PN NGC 6565, which is in quasi-equilibrium, since the stellar evolution slows down and the luminosity gradient rapidly decreases while approaching the white dwarf domain (Paper IV).

In absence of an "evolving" photo-ionization code, which takes into account the gas reactions to a fast changing UV flux, at the moment we apply CLOUDY to the present nebula, (NGC 6818) $\left(t_{0}\right)$, as well to (NGC 6818) $\left(t_{0}-100 \mathrm{yr}\right)$ and (NGC 6818) $\left(t_{0}+100 \mathrm{yr}\right)$. Both the past and the future "snapshots" come from a homologous expansion (i.e. $r \propto t$ and $N(\mathrm{H})$ $\propto t^{-3}$ ), being the dynamical time $t_{0}=2300 \mathrm{yr}$ and $\Delta t<<t_{0}$. Moreover, the evolutionary track of the $0.625 M_{\odot}$ H-burning post-AGB star by Blöcker (1995) was adopted for the stellar parameters. Last, we have selected the quite thin East sector at $\mathrm{PA}=90^{\circ}$ as "mean" density radial profile of (NGC 6818) $\left(t_{0}\right)$.

The input data are contained in Table 5. The results, shown in Fig. 9, can be synthesized as follows:

- at $\left(t_{0}-100 \mathrm{yr}\right)$ (upper row) the intense UV flux of the luminous and hot star completely ionizes the nebula, which appears as a very high excitation object at large $T$ e, optically thin in all the directions;

- at $\left(t_{0}\right)$ (central row) the stellar luminosity is hardly sufficient for ionizing the gas, and the high excitation nebula is almost thick in some directions, where the low ionization emissions emerge;

- at $\left(t_{0}+100 \mathrm{yr}\right)$ (bottom row) the UV flux is far inadequate: the innermost plasma maintains a high degree of excitation, 
but the external layers are thick, with prominent [O I], [O II], [N II] and [S II] lines. Note that the outermost parts of the "steady" photo-ionization model are neutral, whereas are recombining in a "true" evolving nebula (they create a faint halo embedding the main object).

Although these results call for caution due to the photoionization model limitations, nevertheless they support the evolutive scenario of the previous sections: NGC 6818 is a PN at the very beginning of the recombination phase.

A direct confirmation is expected of the detailed analysis of the equatorial moustaches, representing the densest and brightest (i.e. thickest) regions of NGC 6818 (Sect. 5.3). To this end we have first de-projected the spectral images through Eq. (1), and then repeated the complete procedure already applied to the zvpc.

\subsection{Equatorial moustaches}

Let's consider the southern, approaching moustache in PA = $10^{\circ}$. The de-projected radial profiles of the main emissions, the diagnostics and the $\frac{X^{i}}{\mathrm{O}^{++}}$ionic abundances are shown in Fig. 10.

We are particularly interested to $\lambda 6300 \AA$ of [O I], marking the neutral (or almost neutral) nebular layers, where the efficiency of the resonant charge-exchange reaction $\mathrm{O}^{+}+\mathrm{H}^{0} \leftrightarrows$ $\mathrm{O}^{0}+\mathrm{H}^{+}$largely increases (Williams 1973). Note that on the one hand the $[\mathrm{O} \mathrm{I}]$ line is very sensitive to the physical conditions, on the other hand no precise information is yet available for the electron temperature of the external, neutral (or almost neutral) gas dominated by the recombination and cooling processes. We arbitrarily adopt $T$ e(almost neutral gas) $=8000 \mathrm{~K}$, which is lower than $T$ e(ionized gas), but not enough low to compromise the [O I] emissivity (a choice supported by the large kinetic energy of the free electrons in NGC 6818; see Sect. 5.3).

Moreover, referring to the ionization correcting factor ic $f\left(\mathrm{O}^{++}\right)_{\text {outer }}$ of Sect. 6.3, it includes $\mathrm{H}^{0}$, whose contribution comes from the equilibrium condition $\mathrm{O}^{+} / \mathrm{O}^{0} \simeq 0.82 \times$ $\left(\mathrm{H}^{+} / \mathrm{H}^{0}\right)$, valid for $\mathrm{O}^{0}>\mathrm{O}^{+}$, i.e. in the nebular regions affected by the $\mathrm{O}^{+}+\mathrm{H}^{0} \leftrightarrows \mathrm{O}^{0}+\mathrm{H}^{+}$reaction (Williams 1973; Stancil et al. 1999).

The observational results of Fig. 10 must be compared with the "steady" photo-ionization model of the moustache presented in Fig. 11 (the stellar parameters obviously refer to time $t_{0}$ of Table 5).

Besides the general features common to all the directions (i.e. the decreasing $T \mathrm{e}[\mathrm{O} \mathrm{III}]$ radial profile, $T \mathrm{e}[\mathrm{N}$ II $]<$ $T \mathrm{e}[\mathrm{O}$ III $], \mathrm{He}^{++} / \mathrm{O}^{++}=\mathrm{He}^{+} / \mathrm{O}^{++} \simeq 120, \mathrm{Ne}(\mathrm{H} \alpha)=N \mathrm{e}[\mathrm{S}$ II $]$ for $\left(\epsilon_{1} \times r_{\text {cspl }} \times D\right) \simeq 9.5$ arcsec kpc, and so on), the moustache of NGC 6818 (Fig. 10) exhibits a remarkable peculiarity: the external layers are partially neutral, and the outward ionization decline is very smooth, as expected of a recombining region. On the contrary, the "steady" photo-ionization model in Fig. 11 predicts an abrupt ionization fall (to be noticed: the photo-ionization model at $\left(t_{0}-100 \mathrm{yr}\right)$, not shown here, indicates that a century ago the moustache was optically thin to the UV stellar flux).
The Ne depletion rate for recombination is given by:

$$
\mathrm{d} N \mathrm{e} / \mathrm{d} t=-\alpha_{\mathrm{B}} \times N \mathrm{e} \times N\left(\mathrm{H}^{+}\right)
$$

where $\alpha_{\mathrm{B}}$ is the recombination coefficient (Storey \& Hummer 1995). Integrating Eq. (13) and assuming $\mathrm{Ne}=1.15 \times N\left(\mathrm{H}^{+}\right)$, we obtain:

$t=1.15 \times \frac{N \mathrm{e}\left(t_{0}-t\right)-N \mathrm{e}\left(t_{0}\right)}{\alpha_{\mathrm{B}} \times N \mathrm{e}\left(t_{0}-t\right) \times N \mathrm{e}\left(t_{0}\right)}$

which provides the time $t$ elapsed from the beginning of the recombination once are known $\mathrm{Ne}\left(t_{0}\right)$, the present electron density, and $\mathrm{Ne}\left(t_{0}-t\right)$, the electron density at the start of the process.

The application of Eq. (14) to the external parts of the "true" moustache (Fig. 10) furnishes $t=30-60 \mathrm{yr}(t=60-$ $120 \mathrm{yr}$ and 20-40 yr for $T$ e(almost neutral gas $)=5000 \mathrm{~K}$ and $12000 \mathrm{~K}$, respectively). In spite of the heavy assumptions, this agrees with all the previous evidences suggesting that the recombination phase has just begun in NGC 6818.

\section{The 3-D morpho-kinematical structure}

\subsection{General}

The reconstruction of the gas distribution in the nebular slices covered by the slit was introduced in Papers I and II. In the case of NGC 6818 we have selected $\lambda 4686 \AA$ of He II, $\lambda 5007 \AA$ of [O III] and $\lambda 6584 \AA$ of [N II] as representative of the high, mean and low ionization regions, respectively. Note that $\mathrm{H} \alpha$, the marker of the whole ionized gas distribution, cannot be utilized because of the blurred appearance.

The spectral images of the forbidden lines have been deconvolved for seeing, spectral resolution and thermal motions, while also fine structure has been taken into account for $\lambda 4686 \AA$ of He II. They all were de-projected through Eq. (1), and assembled by means of the 3 -D rendering procedure described in Paper III.

In order to reproduce the spatial structure on the paper we adopt the usual method: a series of opaque reconstructions of the nebula seen from different directions, separated by $15^{\circ}$. Each couple forms a stereoscopic pair providing a 3-D view of NGC 6818.

The novelties are represented by the multicolor projection and the movies.

\subsection{Opaque reconstruction}

For reasons of space we only present the opaque reconstruction in He II, [O III] and [N II] for a rotation of $180^{\circ}$ around the East-West axis (close to the minor axis). This is shown in Figs. 12 to 16, where the upper-right panel corresponds to the nebula seen from the Earth (West is up and North to the left, to allow the reader the stereo-view).

The high excitation layers of NGC 6818 (Fig. 12) form an inhomogeneous shell seen almost equatorial on, open-ended at North and South; it merges in a closed ellipsoid at lower $\lambda 4686 \AA$ fluxes. 

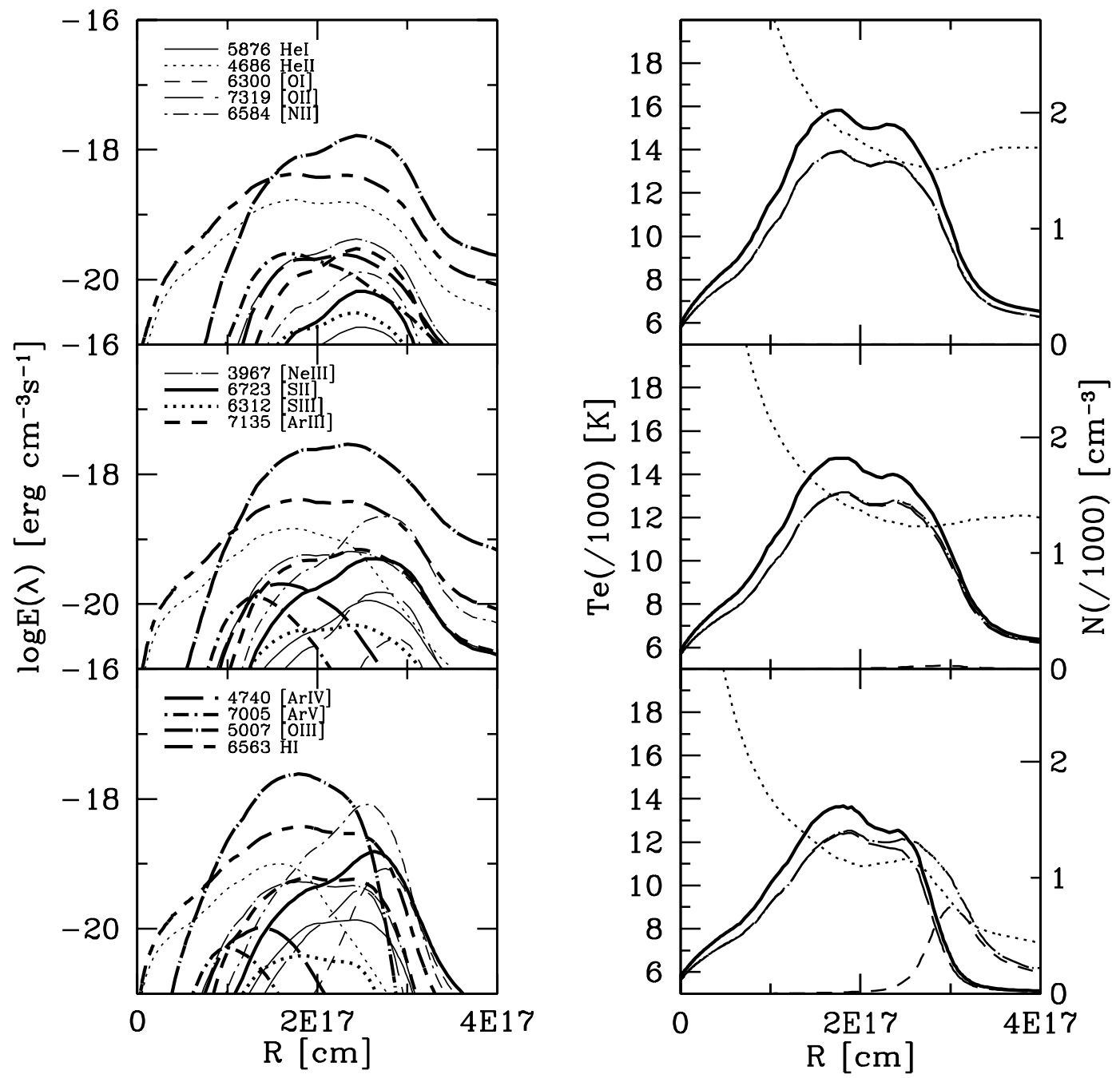

Fig. 9. "Steady" photo-ionization model of NGC 6818, East sector in PA $=90^{\circ}$, at three epochs: $\left(t_{0}-100\right.$ yr) (upper row), $t_{0}$ (central row), and $\left(t_{0}+100 \mathrm{yr}\right)$ (bottom row), $t_{0}=2300 \mathrm{yr}$ being the dynamical nebular age. Left panels: radial profiles of the absolute line fluxes. Right panels: the diagnostics; left ordinate scale: $T$ e (dotted line); right ordinate scale: $N$ e (thick continuous line), $N\left(\mathrm{H}_{\text {tot }}\right)\left(\right.$ dotted-dashed thin line), $N\left(\mathrm{H}^{+}\right)$ (long-dashed thin line) and $N\left(\mathrm{H}^{0}\right)$ (short-dashed thin line).

The opaque reconstruction in [O III] is given for two absolute flux cuts: $\log E(\lambda 5007 \AA)=-17.32 \mathrm{erg} \mathrm{s}^{-1} \mathrm{~cm}^{-3}$ (Fig. 13), and $-17.75 \mathrm{erg} \mathrm{s}^{-1} \mathrm{~cm}^{-3}$ (Fig. 14). Since $\mathrm{O} / \mathrm{H}=$ $5.5 \times 10^{-4} \simeq \mathrm{O}^{++} / \mathrm{H}^{+}$and $\mathrm{Ne}=1.15 \times N\left(\mathrm{H}^{+}\right)$, they correspond to $N \mathrm{e} \simeq 1500 \mathrm{~cm}^{-3}$ (Fig. 13), and $N \mathrm{e} \simeq 900 \mathrm{~cm}^{-3}$ (Fig. 14) (for $T \mathrm{e}=12000 \mathrm{~K}$ and $\epsilon_{1}=1$ ). The [O III] high cut represents the densest regions of the inner shell, mainly constituted by the equatorial moustaches and by an extended cup in the southern part, whereas the external, inhomogeneous shell appears in the low cut frames, characterized by the large hole at North (along the major axis).

The brightest and the faintest [N II] regions refer to $\lambda 6584 \AA$ at the cuts $\log E(\lambda 6584 \AA)=-18.20 \mathrm{erg} \mathrm{s}^{-1} \mathrm{~cm}^{-3}$ (Fig. 15), and $-18.80 \mathrm{erg} \mathrm{s}^{-1} \mathrm{~cm}^{-3}$ (Fig. 16). The [N II] (high cut) distribution mimics the corresponding [O III] one, whereas at lower fluxes the outermost, knotty structure emerges. Note the general weakness of the low ionization layers for $-135^{\circ}<$ $\psi<-45^{\circ}$.

These stereo-reconstructions confirm that NGC 6818 consists of a double shell projected almost equatorial on; the external one is spherical ( $r \simeq 0.090 \mathrm{pc}$ ), faint and patchy. It circumscribes a dense and inhomogeneous tri-axial ellipsoid $(a / 2 \simeq 0.077 \mathrm{pc}, a / b \simeq 1.25, b / c \simeq 1.15)$ characterized by a large hole along the major axis and a couple of thick equatorial regions (the moustaches).

\subsection{Multicolor projection}

A representative sample of the different morphologies assumed by NGC 6818 when changing the line of view is given in Figs. 17 and 18. They show the assembled, multicolor projection at high (He II, blue), medium ([O III], green) and low ([N II], red) ionization for a rotation around the $\mathrm{N}-\mathrm{S}$ axis (close to the major axis; Fig. 17), and around the E-W axis (close to the minor axis; Fig. 18). Also in this case the upper-right panel corresponds to the nebula seen from the Earth (North is up and East to the left), to be compared with Fig. 1, and with the HST multicolor image by A. Hajian \& Y. Terzian at http://ad.usno.navy.mil/pne/gallery.html. 
Table 5. The CLOUDY photo-ionization code: input parameters of the model nebula.

\begin{tabular}{|c|c|}
\hline Radial density profile & right panels of Fig. 9 (cf. Sects. 6.3 and 9) \\
\hline $\begin{array}{l}\text { Chemical abundances: } \\
\mathrm{C}, \mathrm{Na}, \mathrm{Mg}, \mathrm{Si}, \mathrm{Cl}, \mathrm{K}, \mathrm{Ca} \\
\mathrm{He}, \mathrm{N}, \mathrm{O}, \mathrm{Ne}, \mathrm{S}, \mathrm{Ar} \\
\text { other elements }\end{array}$ & $\begin{array}{l}\text { Hyung et al. (1999) } \\
\text { this paper } \\
\text { PN (CLOUDY default) }\end{array}$ \\
\hline Dust & PN (CLOUDY default) \\
\hline Filling factor & 0.5 \\
\hline Exciting star & $\begin{array}{l}\text { at time }\left(t_{0}-100 \mathrm{yr}\right) \text { : blackbody with } T_{*}=170000 \mathrm{~K} \text { and } \log L_{*} / L_{\odot}=3.45(*) \\
\text { at time }\left(t_{0}\right) \text { : blackbody with } T_{*}=160000 \mathrm{~K} \text { and } \log L_{*} / L_{\odot}=3.10(\text { see Sect. } 8) \\
\text { at time }\left(t_{0}+100 \mathrm{yr}\right) \text { : blackbody with } T_{*}=140000 \mathrm{~K} \text { and } \log L_{*} / L_{\odot}=2.60(*)\end{array}$ \\
\hline
\end{tabular}

(*) according to Blöcker (1995).

These "almost" true color reproductions highlight the variety of looks exhibited by the Little Gem: roundish to elliptical, to quasi-bipolar (according to the current morphological classifications; Greig 1972; Stanghellini et al. 1993; Corradi \& Schwarz 1995; Gorny et al. 1997). NGC 6818 resembles NGC 6153, Hu 1-1 and NGC 4071 when seen from $(0,60)$, K 3-57 and M 2-51 from (0,90), A 70 from (90,0), IC 4663 from $(0,30)$ and NGC 7354 from $(150,0)$ (see the imagery catalogues of PNe by Acker et al. 1992; Schwarz et al. 1992; Manchado et al. 1996; Gorny et al. 1999).

\subsection{Movies}

A number of limitations are implicit when showing the 3-D structure on the paper. They are both objective (space, choice of the rotation axes, the nebular parameters, the cuts etc.), and subjective (difficulty in the stereo view). In order to overcome all these handicaps, we have decided to introduce a series of movies as integral and functional part of each PN analysis.

Such an "in fieri" film library, providing the multicolor projection, the opaque recovery in different ions and at various cuts, some slices and radial profiles etc. (suggestions are welcome), can be found at http://web.pd. astro.it/sabbadin (where the acronym "sabbadin" stands for "stratigraphy and best boundary analytic determination in nebulae", sic!).

We are also exploring new graphical solutions, rendering at best the (present) spatial reconstruction and the (forthcoming) spatio-temporal one. In particular, we are sounding the wide potential of the virtual reality.

\section{Discussion and concluding remarks}

In the previous sections of this paper dedicated to the Little Gem we have (partially) extracted and interpreted the huge amount of physical information contained in the ESO NTT+EMMI echellograms by means of the 3-D procedure developed in Papers I to IV for all types of extended, expanding nebulae.

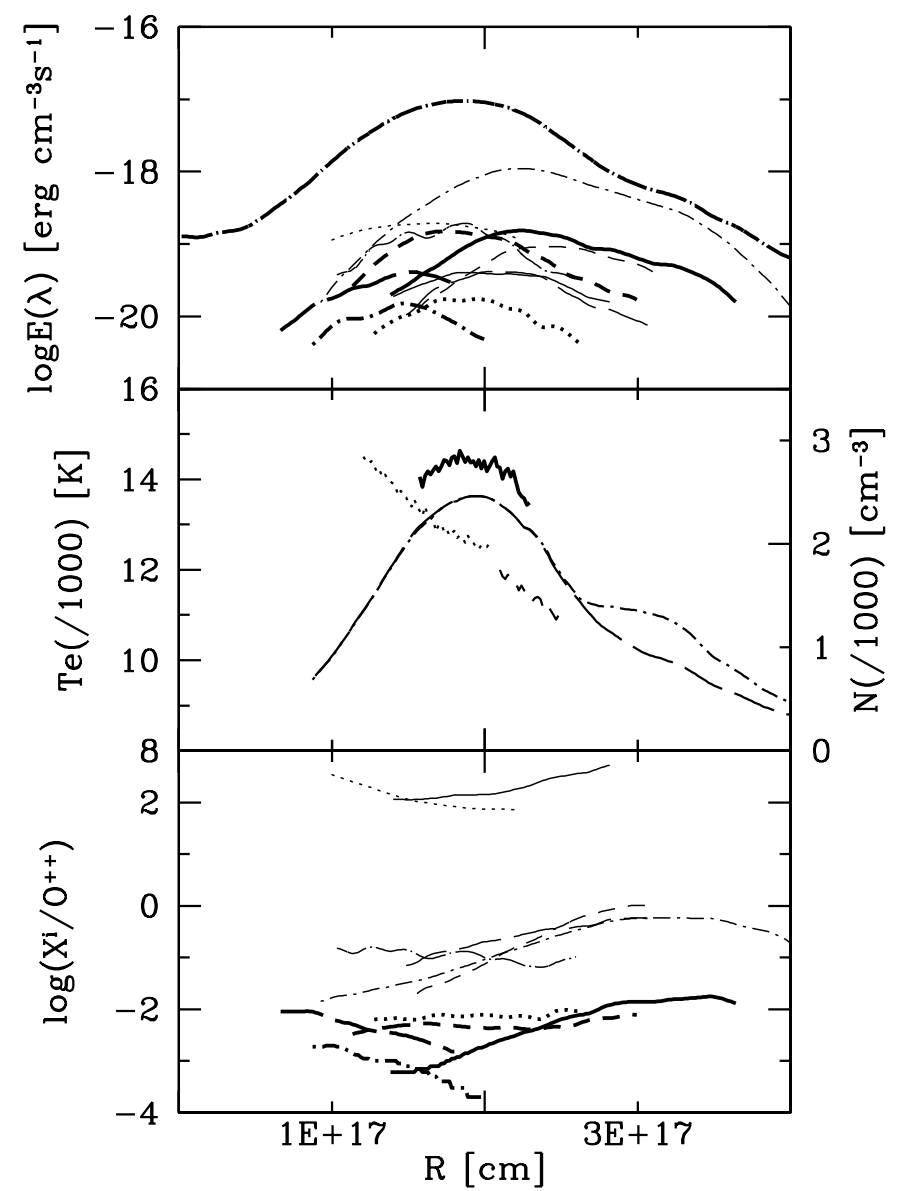

Fig. 10. The observed radial properties of the southern, approaching moustache of NGC 6818 in PA $=10^{\circ}$. Top panel: the absolute flux (in erg $\mathrm{cm}^{-3} \mathrm{~s}^{-1}$ ) of the main emissions; same symbols as Figs. 6 and 9. Middle panel: the diagnostics; left ordinate scale: dotted line $=T \mathrm{e}[\mathrm{O} \mathrm{III}]$, short-dashed line $=T \mathrm{e}[\mathrm{N} \mathrm{II}]$; right ordinate scale: thick continuous line $=N \mathrm{e}[\mathrm{S}$ II $]$, long-dashed line $=N\left(\mathrm{H}^{+}\right)$for $\left(\epsilon_{1} \times r_{\text {cspl }} \times D\right)=9.5$ arcsec kpc, dotted-dashed line $=N\left(\mathrm{H}_{\mathrm{tot}}\right)$. Bottom panel: the $\frac{\mathrm{X}^{i}}{\mathrm{O}^{++}}$ionic abundances (same symbols as the top panel). 


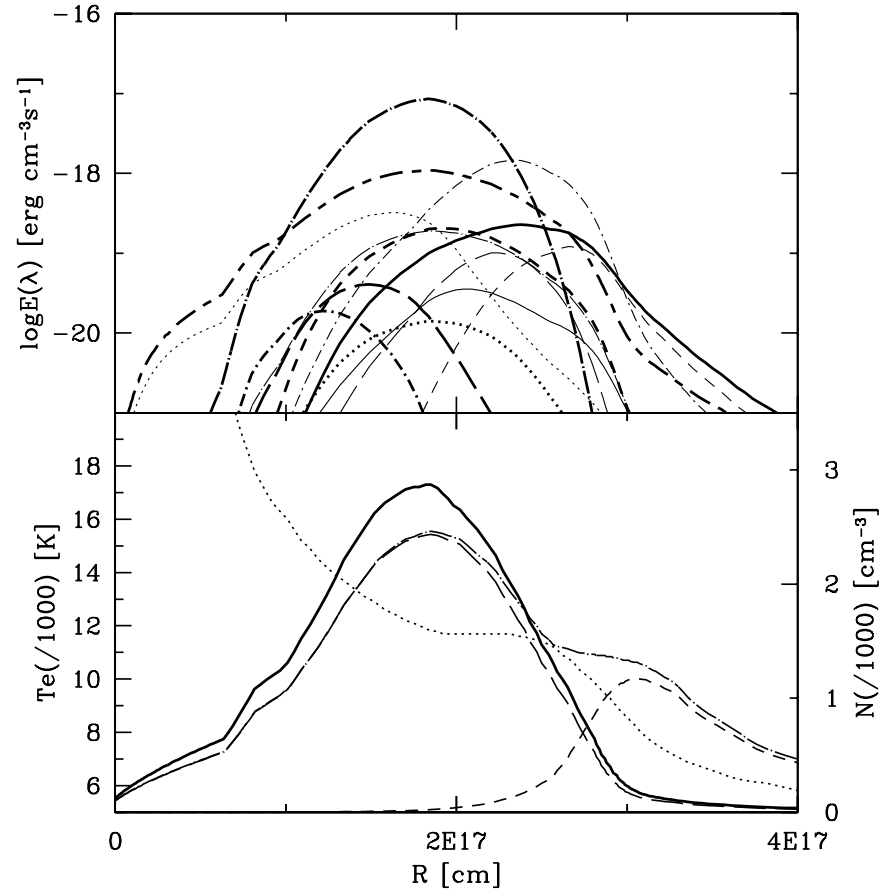

Fig. 11. "Steady" photo-ionization model for the southern, approaching moustache of NGC 6818 in PA $=10^{\circ}$. Top panel: absolute radial flux distribution ( $\mathrm{erg} \mathrm{cm}^{-3} \mathrm{~s}^{-1}$ ) in the different emissions; same symbols as Figs. 6, 9 and 10. Bottom panel: diagnostics radial profile; left ordinate scale: dotted line $=T \mathrm{e}$; right ordinate scale: thick continuous line $=N \mathrm{e}$, long-dashed line $=N\left(\mathrm{H}^{+}\right)$, short-dashed line $=N\left(\mathrm{H}^{0}\right)$, dotted-dashed line $=N\left(\mathrm{H}_{\mathrm{tot}}\right)$.

NGC 6818 results to be a young (3500 yr), optically thin (quasi-thin in some directions) double-shell at a distance of $1.7 \mathrm{kpc}$, projected almost equatorial-on: a tenuous, patchy spheroid ( $r \simeq 0.090 \mathrm{pc}$ ) encircles an inner, dense, inhomogeneous ellipsoid $(a / 2 \simeq 0.077 \mathrm{pc}, a / b \simeq 1.25, b / c \simeq 1.15)$ empty along the major axis and optically thick in a pair of equatorial moustaches.

We recall that outer attached shells have been found in both the 1D and 2D hydrodynamical models (see e.g. Mellema 1995; Corradi et al. 2000; Villaver et al. 2002), due to a Dtype ionization front in the early evolution of the PN. However, all these models fail to reproduce the smooth, innermost radial density profile observed in NGC 6818 (Fig. 7); they predict an empty region (the hot bubble) and a quick density rise (the gas compression is provided by the thermal energy of the hot bubble formed by the adiabatic shock at the interaction region between the high velocity stellar wind and the material ejected during the superwind phase).

The central star of NGC 6818 is a visual binary: a faint, red companion appears at 0.09 arcsec in $\mathrm{PA}=190^{\circ}$, corresponding to a separation $\geq 150 \mathrm{AU}$. For an orbit of low eccentricity the Kepler's third law provides a period $\geq 1500$ yr. Note that (by chance?) the two stellar components appear aligned with the major axis of the nebula.

Despite some pioneering studies, the physical effects produced by a wide binary system on the PN ejection and shaping are still poorly known. Following Soker (1994), an orbital period comparable or longer than the mass-loss episode generating the nebula causes a density enhancement in the equatorial plane and/or spiral structures. Soker (2001) suggests that in wide binary systems (final orbital periods in the range 40 to $10^{4} \mathrm{yr}$ ) an outer, spherical structure is formed by the early AGB wind. Toward the end of the AGB phase, the increased massloss rate creates an accretion disk around the companion. If this blows jets or a collimated fast wind, two lobes appear in the inner nebula (a multi-lobed structure in the case of a precessing accretion disk). Always according to Soker (2001), a fraction of $5 \%$ to $20 \%$ of all PNe originate in such wide binary systems. Curiously, the same author (Soker 1997) includes (with a high degree of confidence) NGC 6818 among the PNe resulting from the common envelope evolution with a sub-stellar companion (planet(s) and/or a brown dwarf). It is evident that the argument deserves further attention.

The Little Gem is in a peculiar evolutionary phase, i.e. at the very beginning of the recombination process. This is caused by the luminosity decline of the $0.625 M_{\odot}$ central star $\left(\log T_{*} \simeq 5.22 \mathrm{~K} ; \log L_{*} / L_{\odot} \simeq 3.1\right)$, which has recently exhausted the nuclear shell burning and is rapidly moving towards the white dwarf region. The stellar drop being fated to continue, NGC 6818 will become thicker and thicker, and the amount of neutral, dusty gas in the outermost layers will increase with time. The ionization front will re-grow only in some hundreds years, when the gas dilution due to the expansion will overcome the slower and slower luminosity decline.

Concerning the observational analogies between NGC 6818 (this paper) and NGC 6565 (Paper IV), in Sect. 8 we have pointed out the probable evolutive contiguity of the two nebulae. We stress here a more facet of the affair, e.g. the importance of the temporal factor: thanks to the excellent spatial and spectral resolutions achieved by the 3-D analysis applied to high quality spectra, we can no longer regard a PN as a static, uniform and un-changeable object; it is a dynamical, inhomogeneous and evolving plasma.

Ironically, this quite reverses the gap between theory and practice outlined in the Introduction: now the existing "steady" photo-ionization models appear inadequate to interprete the observational data. A 3-D "evolving" code is highly desired, providing for the gas reactions to the changing UV flux of the central star.

In summary: we have inferred a self-consistent picture of the Little Gem by means of ESO NTT+EMMI echellograms. Deeper observations at even higher spatial and spectral resolutions will disentangle the still unresolved problems, like the accurate $T \mathrm{e}[\mathrm{N} \mathrm{II}]$ and $\mathrm{Ne}[\mathrm{S} \mathrm{II}]$ radial profiles (Sect. 5), the intriguing ionization structure of the cometary knot in $\mathrm{PA}=150^{\circ}$ (Sect. 4) and the possible blowing of the gas in the Northern and Southern holes (Sect. 4). Moreover, a gradual change of the $[\mathrm{N} \mathrm{II}] /[\mathrm{O} \mathrm{III}]$ morphology is expected in the future HST imagery, due to the peculiar evolutionary phase of the nebula. Concerning the exciting star, a painstaking search in the worldwide archives (both spectroscopic and photometric), and new, deep, UV to IR spectra of the stellar system (hot central star + cold companion) are needed.

At last, the "vexata quaestio": which are the mechanisms and the physical processes ejecting and shaping a PN like 


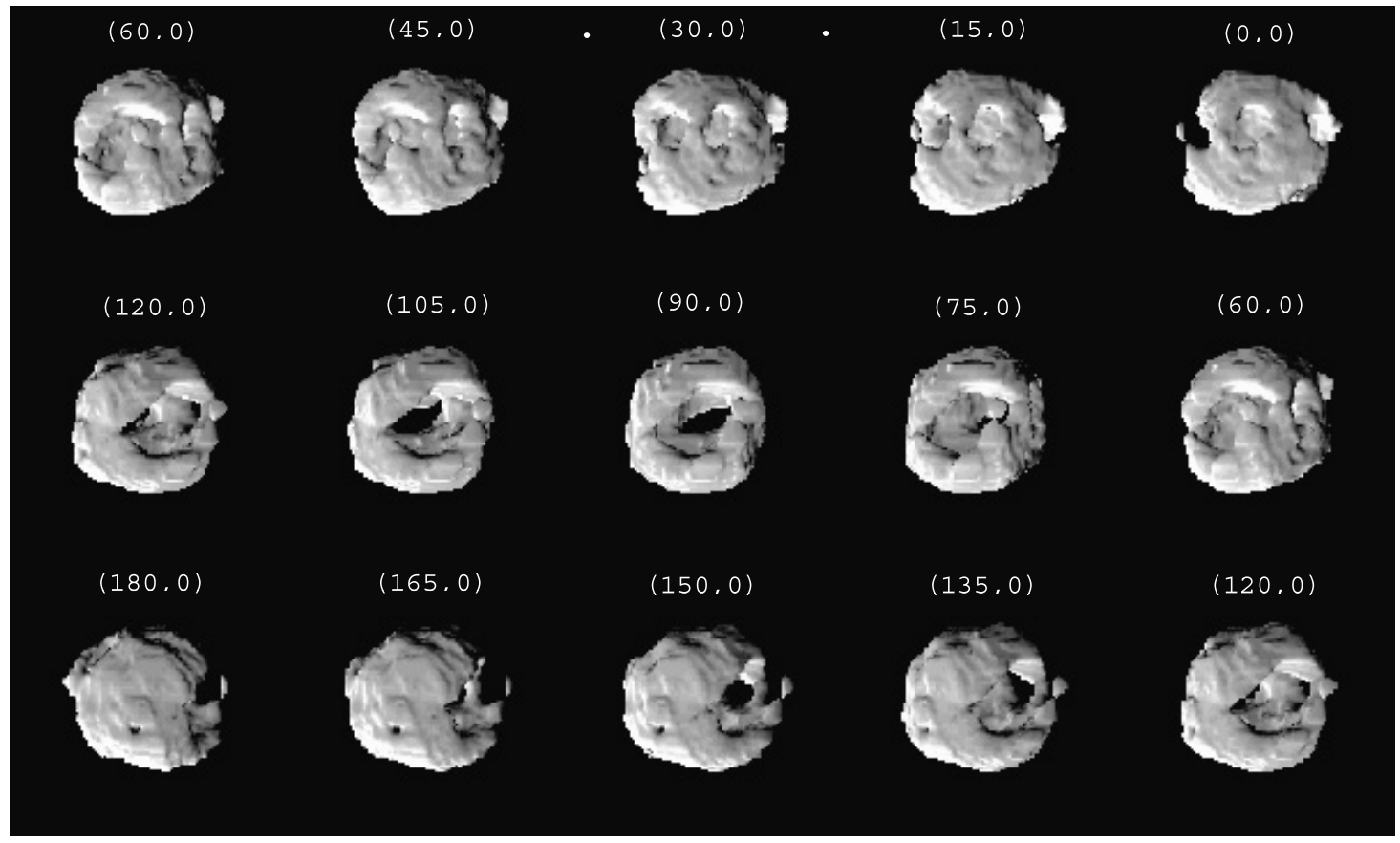

Fig. 12. The structure of NGC 6818 for a rotation around the East-West axis centered on the exciting star. Opaque reconstruction at $\lambda 4686 \AA$ of He II, seen from 13 directions separated by $15^{\circ}$. The line of view is given by $(\theta, \psi)$, where $\theta$ is the zenith angle and $\psi$ the azimuthal angle. Each horizontal couple represents a "direct" stereoscopic pair, and the whole figure provides 12 3-D views of the nebula in as many directions, covering a straight angle. Following Paper III: to obtain the three-dimensional vision, look at a distant object and slowly insert the figure in the field of view, always maintaining your eyes parallel. Alternatively, you can use the two small dots in the upper part of the figure as follows: approach the page till the two dots merge (they appear out of focus); then recede very slowly, always maintaining the two dots superimposed, till the image appears in focus. The upper-right image is the rebuilt-nebula seen from the Earth (West is up and North to the left, to allow the reader the stereo-view).

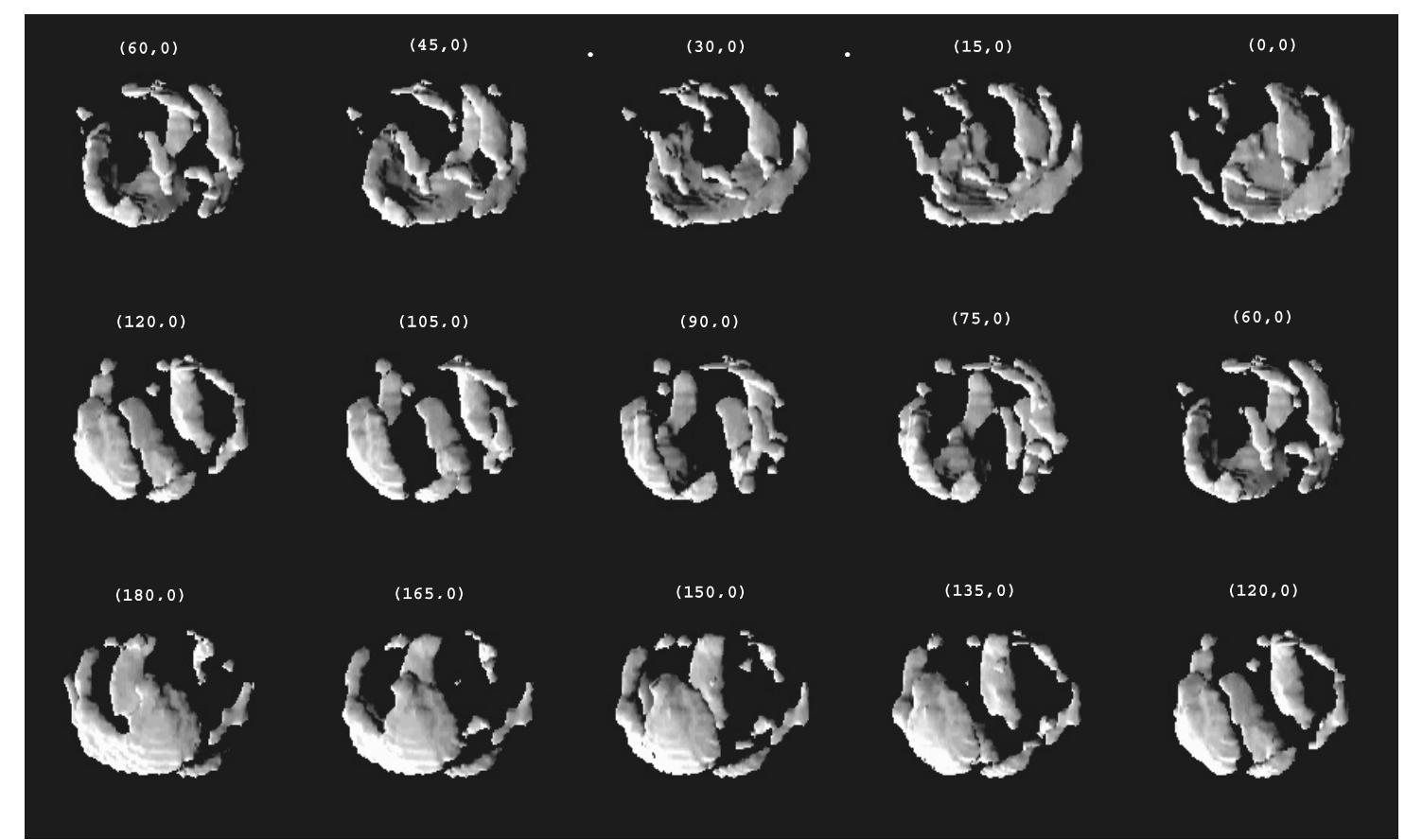

Fig. 13. Same as Fig. 12, but for $[\mathrm{O} \mathrm{III}]$ at the flux cut $\log E(\lambda 5007 \AA)=-17.32 \mathrm{erg} \mathrm{s}^{-1} \mathrm{~cm}^{-3}\left(N \mathrm{e} \simeq 1500 \mathrm{~cm}^{-3}\right.$ for $T \mathrm{e}=12000 \mathrm{~K}$ and $\left.\epsilon_{\mathrm{l}}=1\right)$.

NGC 6818? In our opinion the question appears premature, and the answer is beyond the aims of the paper, given the "forest" of proposed models (see Icke et al. 1992; Mellema 1997;
Dwarkadas \& Balick 1998; Garcia-Segura et al. 1999; Frank 1999; Blackman et al. 2001; Soker \& Rappaport 2001; Balick \& Frank 2002), and the "desert" of carefully studied true 


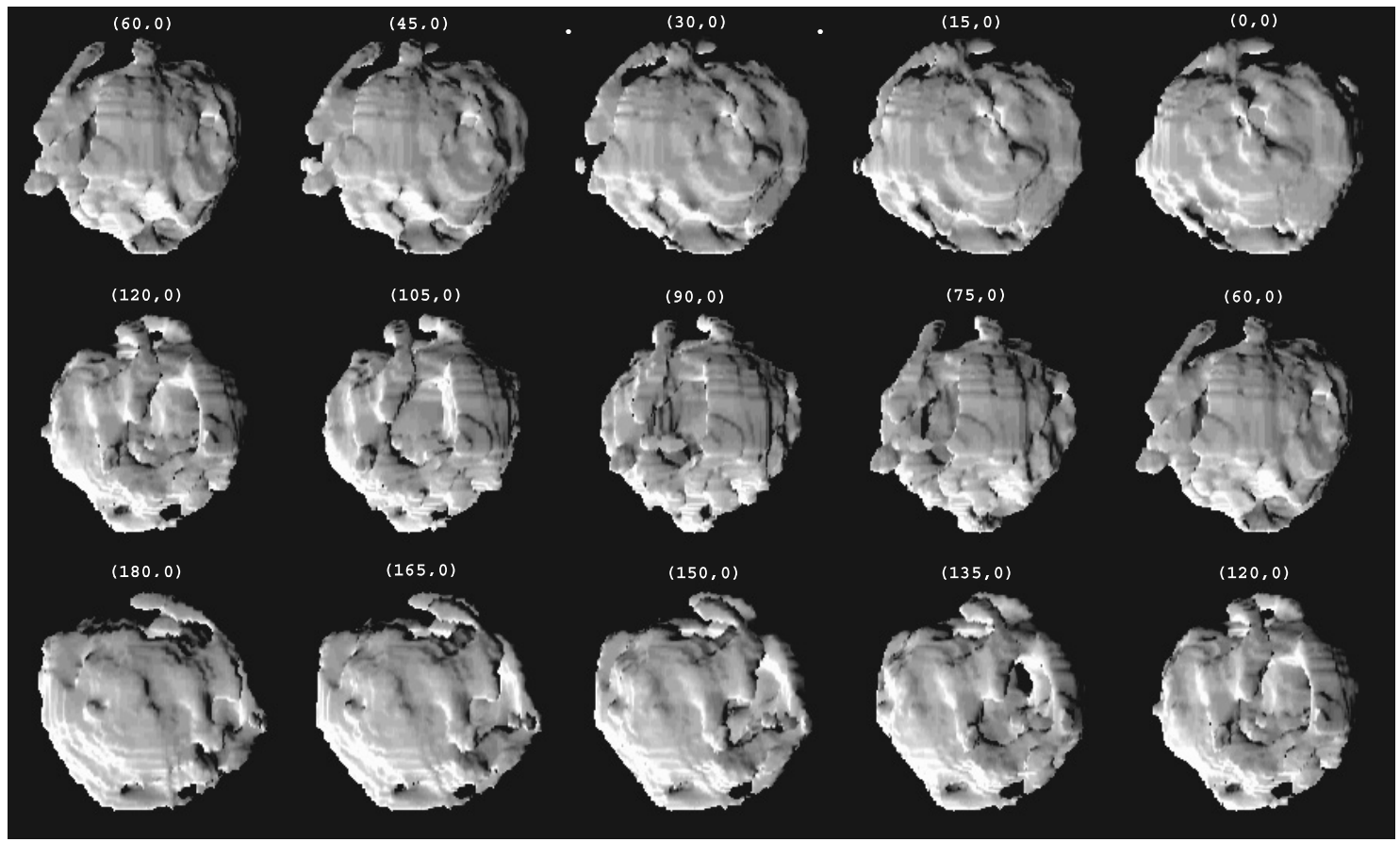

Fig. 14. Same as Fig. 13, but for the [O III] flux cut $\log E(\lambda 5007 \AA)=-17.75 \mathrm{erg} \mathrm{s}^{-1} \mathrm{~cm}^{-3}\left(N \mathrm{e} \simeq 900 \mathrm{~cm}^{-3}\right.$ for $T \mathrm{e}=12000 \mathrm{~K}$ and $\left.\epsilon_{1}=1\right)$.

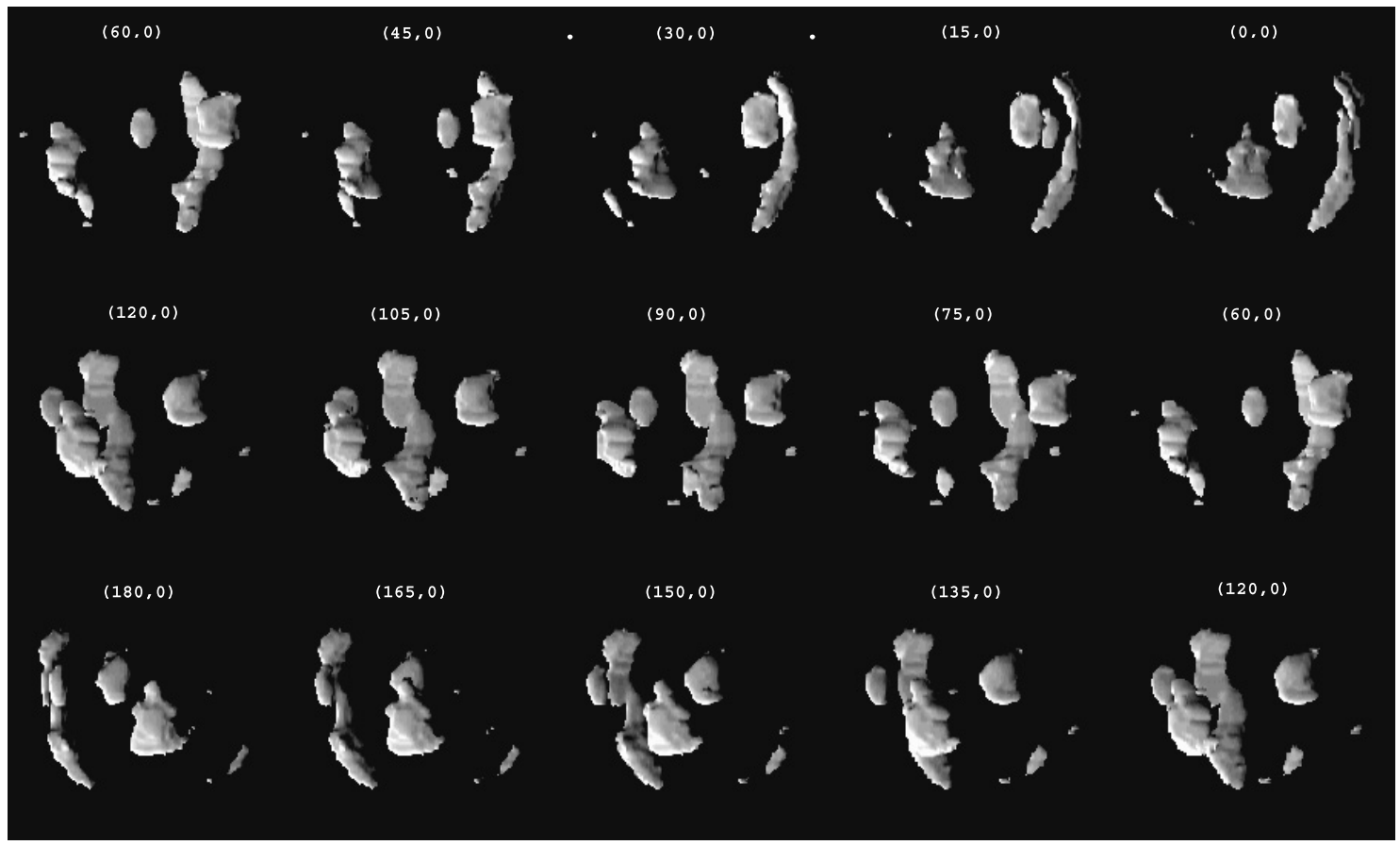

Fig. 15. Same as Figs. 12 to 14 , but for $\left[\mathrm{N}\right.$ II] at the flux cut $\log E(\lambda 6584 \AA)=-18.20 \mathrm{erg} \mathrm{s}^{-1} \mathrm{~cm}^{-3}$ (bright low ionization layers).

nebulae. We are confident that new, reliable and deep insights on each object and on the whole class will come out of the comparative analysis of a representative sample of $\mathrm{PNe}$ and protoPNe.

This is the final goal of our survey carried out with ESO NTT+EMMI and TNG+SARG. Indeed, the superb quality of these echellograms constitutes a powerful tool for unveiling the evolutional secrets of the PNe (as well for masking the cultural gaps of the authors).
Acknowledgements. It is a pleasure to thank Gary Ferland, Arsen Hajian, Garrelt Mellema (the referee), Detlef Schönberner and Noam Soker for their suggestions, comments, encouragements and criticisms.

This paper has been financied by the grant Cofin MM02905817 of the Italian Ministry of Education (MIUR) and partially supported by the grant ASI (Agenzia Spaziale Italiana) I/R/70/00. 


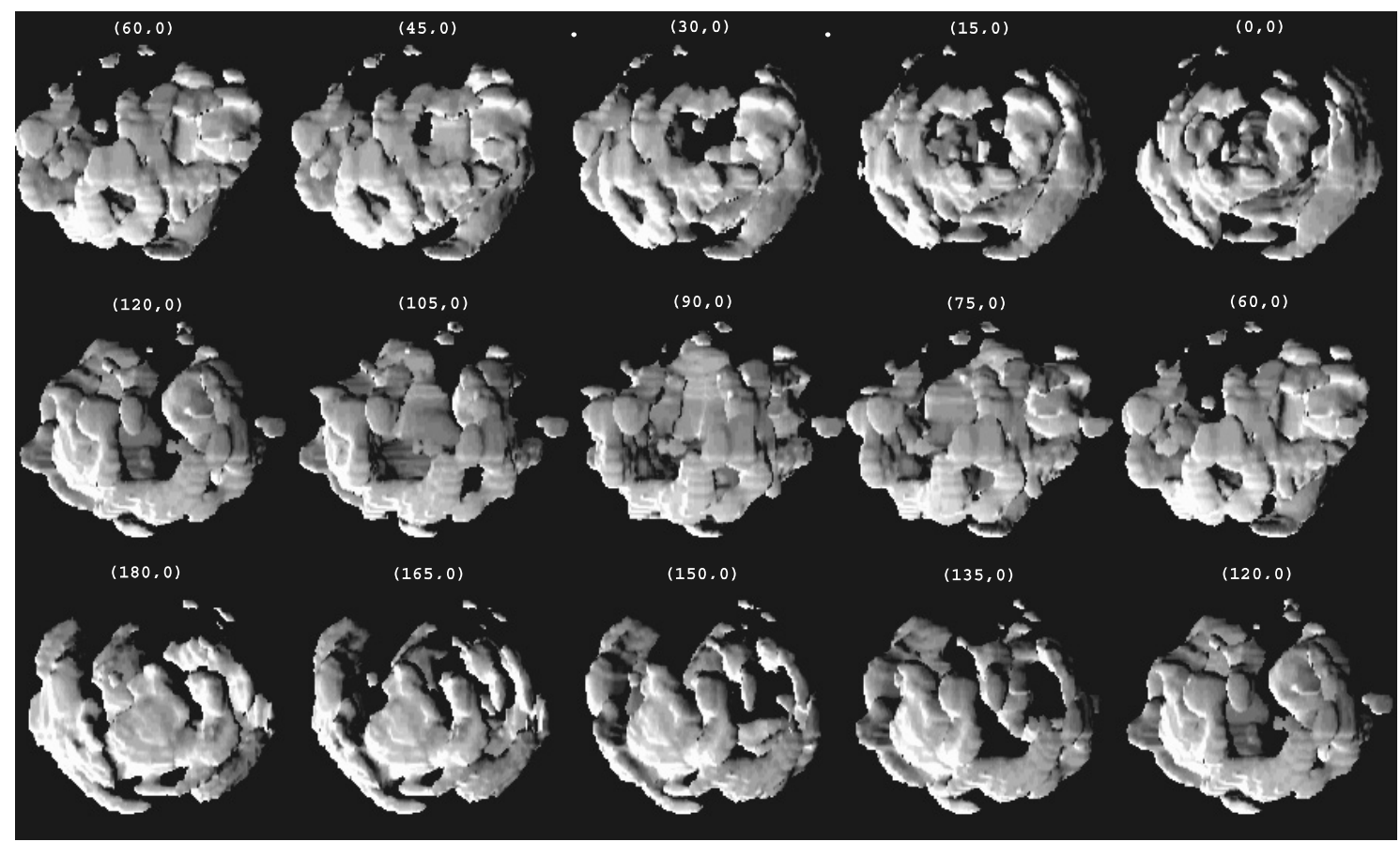

Fig. 16. Same as Fig. 15, but for the [N II] flux cut $\log E(\lambda 6584 \AA)=-18.80 \mathrm{erg} \mathrm{s}^{-1} \mathrm{~cm}^{-3}$ (faint low ionization layers).

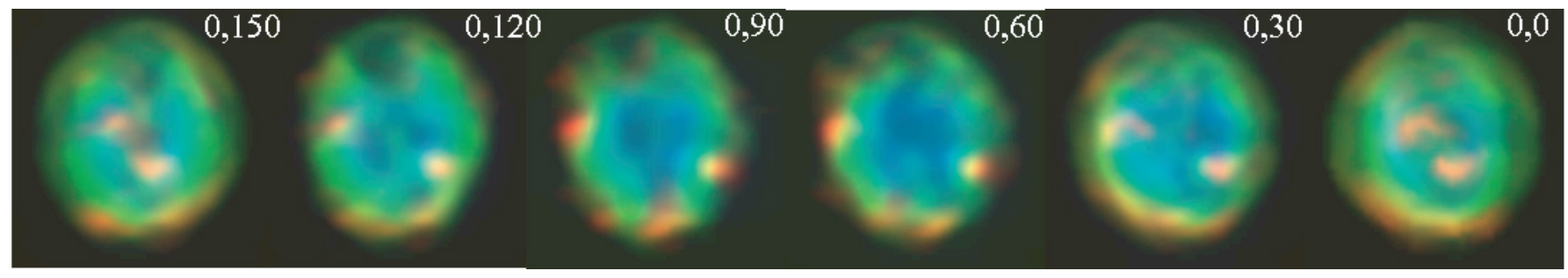

Fig. 17. Multicolor appearance of NGC 6818 (blue $=\mathrm{He}$ II, green $=[\mathrm{O} \mathrm{III}]$, red $=[\mathrm{N} \mathrm{II}]$ ) for a rotation around the N-S axis centered on the exciting star. The right panel, $(0,0)$, corresponds to the re-built nebula seen from the Earth (North is up and East to the left). Same scale as Figs. 12 to 16 . Recall that projection $(\theta, \psi)=$ projection $\left(\theta \pm 180^{\circ}, \psi \pm 180^{\circ}\right)$.

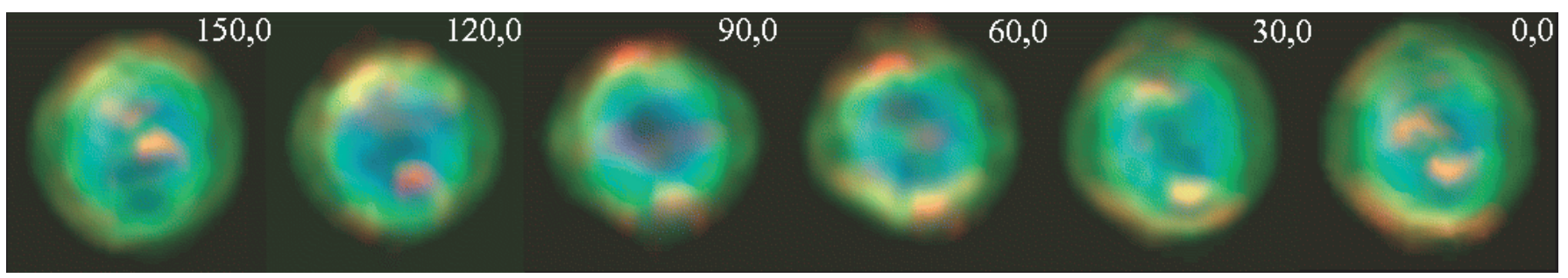

Fig. 18. Same as Fig. 17, but for a rotation around the E-W axis.

\section{References}

Acker, A. 1978, A\&AS, 33, 367

Acker, A., Ochsenbein, F., Stenholm, et al. 1992, Strasbourg-ESO Catalogue of Galactic Planetary Nebulae, (Garching: ESO)

Acker, A., Raytchev, B., Stenholm, B., \& Tylenda, R. 1991, A\&AS, 90,89

Alexander, J., \& Balick, B. 1997, AJ, 114, 713

Aller, L. H. 1984, Physics of Gaseous Nebulae (Dordrecht: Reidel)
Aller, L. H. 1990, PASP, 102, 1097

Aller, L. H. 1994, ApJ, 432, 427

Aller, L. H., \& Czyzak, S. J. 1979, Ap\&SS, 62, 397

Aller, L. H., \& Czyzak, S. J. 1983, ApJS, 51, 211

Amnuel, P. R., Guseinov, O. K., Novruzova, et al. 1984, Ap\&SS, 107, 19

Baker, J. G., \& Menzel, D. H. 1938, ApJ, 88, 52

Balick, B., \& Frank, A. 2002, ARA\&A, 40, 439 
Balick, B., Rugers, M., Terzian, Y., et al. 1993, ApJ, 411, 778

Barker, T. 1983, ApJ, 267, 630

Barker, T. 1986, ApJ, 308, 314

Bensby, T., \& Lundström, I. 2001, A\&A, 374, 599

Berman, L. 1937, LickOB, 487, 47

Bianchi, L., Catanzaro, G., Scuderi, S., \& Hutchings, J. B. 2001, PASP, 113, 697

Blackman, E. G., Frank, A., \& Welch, C. 2001, ApJ, 546, 288

Blöcker, T. 1995, ApJ, 371, 217

Blöcker, T., \& Schönberner, D. 1990, A\&A, 240, L11

Brocklehurst, M. 1971, MNRAS, 153, 471

Cahn, J. H. 1976, AJ, 81, 407

Cahn, J. H., \& Kaler, J. B. 1971, ApJS, 22, 319

Cahn, J. H., Kaler, J. B., \& Stanghellini, L. 1992, A\&AS, 94, 399

Cazetta, J. O., \& Maciel, W. J. 2000, RMxAA, 36, 3

Chengalur, J. N., Lewis, B. M., Eder, J., \& Terzian, Y. 1993, ApJS, 89, 189

Collins, G. W., Daub, C. T., \& O’Dell, C. R. 1961, ApJ, 133, 471

Condon, J. J., Kaplan, D. L., \& Terzian, Y. 1999, ApJS, 123, 219

Corradi, R. L. M., Goncalves, D. R., Villaver, E., et al. 2000, A\&A, 542,861

Corradi, R. L. M., Manso, R., Mampaso, A., \& Schwarz, H. E. 1996, A\&A, 313, 913

Corradi, R. L. M., \& Schwarz, H. E. 1995, A\&A, 293, 871

Cudworth, K. M. 1974, AJ, 79, 1384

Curtis, H. D. 1918, Publ. Lick Obs., 13, 55

Daub, C. T. 1982, ApJ, 260, 612

David, P., Le Squeren, A. M., \& Sivagnanam, P. 1993, A\&A, 277, 474

de Freitas Pacheco, J. A., Maciel, W. J., Costa, R. D. D., \& Barbuy, B. 1991, A\&A, 250, 159

Dopita, M. A., Lawrence, C. J., Ford, H. C., \& Webster, B. L. 1985, ApJ, 296, 390

Dwarkadas, V. V., \& Balick, B. 1998, ApJ, 497, 267

Feibelman, W. A. 1994, PASP, 106, 56

Ferland, G. J., Korista, K. T., Verner, D. A., et al. 1998, PASP, 110, 761

Frank, A. 1999, NewAR, 43, 31

Fruchter, A. S., \& Hook, R. N. 2002, PASP, 114, 144

Gallart, C., Aparicio, A., \& Vilchez, J. R. 1996, AJ, 112, 1928

Garcia-Segura, G., Langer, N., Rozyczka, M., \& Franco, J. 1999, ApJ, 517,767

Gathier, R., \& Pottasch, S. R. 1988, A\&A, 197, 266

Gathier, R., Pottasch, S. R., \& Pel, J. W. 1986, A\&A, 157, 171

Gorny, S. K., Schwarz, H. E., Corradi, R. L. M., \& Van Winckel, H. 1999, A\&AS, 136, 145

Gorny, S. K., Stasinska, G., \& Tylenda, R. 1997, A\&A, 318, 256

Greig, W. E. 1972, A\&A, 18, 70

Gruenwald, R., \& Viegas, S. M. 1995, A\&A, 303, 535

Gurzadyan, G. A. 1970, Planetary nebulae (Dordrecht: D. Reidel)

Habing, H. J. 1996, A\&ARv, 7, 97

Harman, R. F., \& Seaton, M. J. 1966, MNRAS, 132, 15

Harrington, J. P. 1989, in Planetary Nebulae, ed. S. Torres-Peimbert, IAU Symp., 131, 157

Hummer, D. G., \& Storey, P. J. 1987, MNRAS, 224, 801

Hyung, S., Aller, L. H., \& Feibelman, W. A. 1999, ApJ, 514, 878

Iben, I. Jr. 1984, ApJ, 277, 333

Icke, V., Balick, B., \& Frank, A. 1992, A\&A, 253, 224

Jacoby, G. H., \& Kaler, J. B. 1989, AJ, 98, 1662

Kaler, J. B. 1986, ApJ, 308, 337

Keenan, F. P., Aller, L. H., Bell, K. L., et al. 1996, MNRAS, 281, 1073

Keenan, F. P., Aller, L. H., Bell, K. L., et al. 1999, MNRAS, 304, 27

Kingsburgh, R. L., \& Barlow, M. J. 1992, MNRAS, 257, 317

Kohoutek, L., \& Martin, W. 1981, A\&AS, 44, 325

Kovacs, G. 2000, A\&A, 363, L1
Liu, X. -W., \& Danziger, I. J. 1993, MNRAS, 263, 256

Lutz, J. H. 1973, ApJ, 181, 135

Maciel, W. J. 1984, A\&AS, 55, 253

Maciel, W. J., \& Pottasch, S. R. 1980, A\&A, 88, 1

Mal'kov, Yu. F. 1997, ARep, 41, 760

Manchado, A., Guerrero, M. A., Stanghellini, L., \& Serra-Ricart, M. 1996, The IAC Morphological Catalog of Northern Planetary Nebulae, IAC

Marten, H., \& Szczerba, R. 1997, A\&A, 325, 1132

Martin, W. 1981, A\&A, 98, 328

Mathis, J. S., Torres-Peimbert, S., \& Peimbert, M. 1998, ApJ, 495, 328

McKenna, F. C., Keenan, F. P., Kaler, J. B., et al. 1996, PASP, 108, 610

Meatheringham, S. J., Wood, P. R., \& Faulkner, D. J. 1988, ApJ, 334, 862

Mellema, G. 1995, MNRAS, 277, 173

Mellema, G. 1997, A\&A, 321, L29

Milne, D. K., \& Aller, L. H. 1975, A\&A, 38, 183

O'Dell, C. R. 1962, ApJ, 135, 371

Osterbrock, D. E. 1989, Astrophysics of Gaseous Nebulae and Active Galactic Nuclei, Mill Valley, CA Univ. Sci.

Paczynski, B. 1970, AcA, 20, 47

Palen, S., Balick, B., Hajian, A. R., et al. 2002, AJ, 123, 2666

Palmer, H. K. 1903, ApJ, 18, 218

Pease, F. G. 1917, ApJ, 46, 24

Peimbert, M., \& Torres-Peimbert, S. 1983, in Planetary Nebulae, ed. D. R. Flower, IAU Symp., 103, 233

Pequignot, D. 1997, in Planetary Nebulae, ed. H. J. Habing, \& H. J. G. L. M. Lamers, IAU Symp., 180, 167

Phillips, J. P. 2002, ApJS, 139, 199

Phillips, J. P., \& Pottasch, S. R. 1984, A\&A, 130, 91

Pottasch, S. R. 1984, Planetary Nebulae, a Study of Late Stages of Stellar Evolution (Dordrecht: Reidel)

Pottasch, S. R., \& Preite-Martinez, A. 1983, A\&A, 126, 31

Preite-Martinez, A., Acker, A., Köppen, J., \& Stenholm, B. 1989, A\&AS, 81, 309

Ragazzoni, R., Cappellaro, E., Benetti, S., et al. 2001, A\&A, 369, 1088 (Paper III)

Reed, D. S., Balick, B., Hajian, A. R., et al. 1999, AJ, 118, 2430

Rowlands, N., Houck, J. R., Herter, T., et al. 1989, ApJ, 341, 901

Rubin, R. H., Dufour, R. J., Browning, M., \& Harrington, J. P. 1998, AAS, 192.5319

Sabbadin, F. 1984, MNRAS, 210, 341

Sabbadin, F., Benetti, S., Cappellaro, E., \& Turatto, M. 2000b, A\&A, 361, 1112 (Paper II)

Sabbadin, F., Bianchini, A., Ortolani, S., \& Strafella, F. 1985, MNRAS, 217, 539

Sabbadin, F., Cappellaro, E., Benetti, S., et al. 2000a, A\&A, 355, 688 (Paper I)

Sabbadin, F., Cappellaro, E., \& Turatto, M. 1987, A\&A, 182, 305

Saurer, W. 1995, A\&A, 297, 261

Schönberner, D. 1981, A\&A, 103, 119

Schönberner, D. 1983, ApJ, 272, 708

Schwarz, H. E., Corradi, R. L. M., \& Melnick, J. 1992, A\&AS, 96, 23

Seaton, M. J. 1968, MNRAS, 139, 129

Seaton, M. J. 1979, MNRAS, 187, 73P

Shaw, R. A., \& Kaler, J. B. 1985, ApJ, 295, 537

Shields, G. A., Aller, L. H., Keyes, C. D., \& Czyzak, S. J. 1981, ApJ, 248,569

Shklovski, I. S. 1956, AZh, 33, 315

Sjouwerman, L. O., van Langevelde, H. J., Winnberg, A., \& Habing, H. J. 1998, A\&AS, 128, 35 
Soker, N. 1994, MNRAS, 270, 774

Soker, N. 1997, ApJS, 112, 487

Soker, N. 2001, ApJ, 558, 157

Soker, N., \& Rappaport, S. 2001, ApJ, 557, 256

Stancil, P. C., Schultz, D. R., Kimura, M., et al. 1999, A\&AS, 140, 225

Stanghellini, L., Corradi, R. L. M., \& Schwarz, H. E. 1993, A\&A, 276, 463

Storey, P. J., \& Hummer, D. G. 1995, MNRAS, 272, 41

Turatto, M., Cappellaro, E., Ragazzoni, R., et al. 2002, A\&A, 384, 1062 (Paper IV)

Tylenda, R. 1986, A\&A, 156, 217
Tylenda, R., Acker, A., \& Stenholm, B. 1993, A\&AS, 102, 595

Tylenda, R., Acker, A., Stenholm, B., \& Köppen, J. 1992, A\&AS, 95, 337

van de Steene, G. C., \& Zijlstra, A. A. 1994, A\&AS, 108, 485

Vassiliadis, E., \& Wood, P. R. 1994, ApJS, 92, 125

Villaver, E., Manchado, A., \& Garcia-Segura, G. 2002, ApJ, 581, 1204

Webster, B. J. 1983, PASP, 95, 610

Weedman, D. W. 1968, ApJ, 153, 49

Williams, R. E. 1973, MNRAS, 164, 111

Wilson, O. C. 1950, ApJ, 111, 279

Wood, P. R., \& Faulkner, D. J. 1986, ApJ, 307, 659

Zhang, C. Y. 1995, ApJS, 98, 659 